\title{
Mixing and flow-induced silk fibroin self-assembly in microfluidic and semi-batch nanoprecipitation
}

Saphia A. L. Matthew, ${ }^{\dagger}$ Refaya Rezwan ${ }^{\dagger}$, Yvonne Perrie $e^{\dagger}$ and F. Philipp Seib ${ }^{*}+, \S, \$$

* Corresponding author: Tel: +44 (0) 141548 2510; E-mail: philipp.seib@strath.ac.uk.

$\dagger$ Strathclyde Institute of Pharmacy and Biomedical Sciences, University of Strathclyde, 161 Cathedral Street, Glasgow, G4 0RE, U.K.

$\$$ Department of Pharmacy, ASA University Bangladesh, 23/3 Bir Uttam A.N.M Nuruzzaman Sarak, Dhaka 1207, Bangladesh.

$\S$ EPSRC Future Manufacturing Research Hub for Continuous Manufacturing and Advanced Crystallisation (CMAC), University of Strathclyde, Technology and Innovation Centre, 99 George Street, Glasgow G1 1RD, U.K.

\$ Leibniz Institute of Polymer Research Dresden, Max Bergmann Center of Biomaterials Dresden, Hohe Strasse 6, 01069 Dresden, Germany.

KEYWORDS silk fibroin, nanoprecipitation, self-assembly, nanoparticle, biopolymer.

For Table of Contents Use Only. 


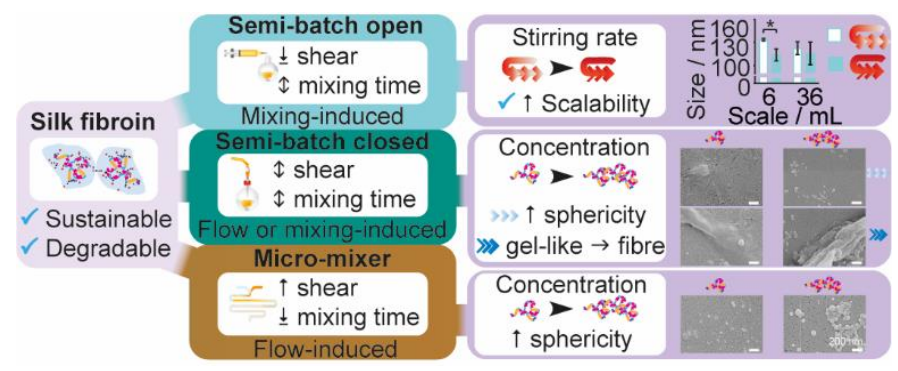

\begin{abstract}
Here, we report the modulation of silk fibroin self-assembly by varying factors which control shear and mixing during nanoprecipitation in semi-batch and micro-mixers. For feeds processed at low shear in a semi-batch format, the properties of secondary assemblies (nanoparticles) were scalable by reducing the mixing time by stirring $(0<400 \mathrm{rpm})$. For low mixing times, moving from low to high shear processing increased the extent of self-assembly $\left(0.017<16.96 \mathrm{~mL} \mathrm{~min}^{-1}\right)$ for $0.5,2$ and $3 \% \mathrm{w} / \mathrm{v}$ silk. In high shear regimes, the size and polydispersity index of assemblies decreased with mixing time, as stirring rate $(800,400<0 \mathrm{rpm})$ and feed addition height $(3.5<0 \mathrm{~cm})$ increased. Finally, in conditions of high shear and low mixing time, the feed concentration controlled the assembly shape, size, and polydispersity index in microfluidic $(0.5,3.0<2 \% \mathrm{w} / \mathrm{v})$ and semi-batch format $(3.0<0.5 \% \mathrm{w} / \mathrm{v})$. This work provides new insight into the manufacture of low polydispersity, spherical and worm-like silk nanoparticles.
\end{abstract}

\title{
1. INTRODUCTION
}

Silk fibroin produced by the Bombyx mori silkworm is increasingly proposed for a range of drug delivery applications, ${ }^{1}$ as this biopolymer exhibits several favorable characteristics, including biocompatibility and biodegradability, ${ }^{2-4}$ and a number of products have been translated to the clinic. $^{5}$ A variety of material types ${ }^{5}$ and crystallinities $^{5-8}$ can also be accessed from the reverseengineered silk solution, as the block copolymer primary structure can exist in a range of 
polymorphic states, notably silk I-II. ${ }^{9}$ The silk I polymorph has a high composition of $\beta$-turns, helices and random coils which bestow aqueous solubility. This metastable solution is found in the silkworm gland or is obtained by regeneration of the degummed silkworm cocoon. ${ }^{10}$

The rate of structural conversion of aqueous silk I to the more thermodynamically stable and solid silk II structure can be increased by displacement of the protein hydration layer with a watermiscible organic solvent ${ }^{11-13}$ and the application of physical shear forces under flow. ${ }^{10}$ The intermolecular hydrogen-bonding ability of silk molecules under shear flow enables the spinning of liquid silk dope at ambient conditions and remarkably little work input. ${ }^{14}$ Similarly, this fundamental property determines the outcome of high shear fluid processing of the regenerated aqueous solutions into structures such as nanoparticles.

The physicochemical properties of nanoparticles affect performance factors, including drug loading, stability on storage and in vivo performance. ${ }^{15}$ Favorable attributes for drug carriers include nanoparticle sizes between 100 and $200 \mathrm{~nm}$ and a hydrophilic and negatively charged shell. ${ }^{16}$ The micelle-like hydrophobic core and anionic, hydrophilic shell structure ${ }^{10,17,18}$ of silk nanoparticles impart hemocompatibility ex vivo ${ }^{3}$ and enable post-synthetic, electrostatic drug loading. ${ }^{1,11,19,20}$ The polar amino acids, such as tyrosine, serine, histidine and lysine, on the nanoparticle surface are also amenable to covalent modification ${ }^{3}$ and have enabled conjugation of cell-targeting agents (e.g. folate $\left.{ }^{21}\right)$ and implementation of stealth technology, such as PEGylation, to suppress various processes, such as agglomeration in physiological media, ${ }^{3}$ blood platelet coagulation ex vivo, ${ }^{3,22}$ and proinflammatory responses in vitro. ${ }^{3,23}$

Silk particles of sub-micron size $(25-200 \mathrm{~nm})$ are suitable for drug delivery and can be manufactured by eight major methods (reviewed previously ${ }^{24}$ ): capillary microdot printing, ${ }^{25}$ desolvation,,${ }^{11,13,20,22}$ supercritical $\mathrm{CO}_{2},{ }^{26}$ electrospraying, ${ }^{27}$ emulsification, ${ }^{28}$ ionic liquid 
dissolution, ${ }^{4}$ milling ${ }^{29}$ and electrogelation. ${ }^{30}$ Of these methods, desolvation is an accessible, lowenergy-expenditure nanoprecipitation process which has been used to tune the key quality attributes of protein ${ }^{12,13,31}$ and polymeric ${ }^{32}$ nanoparticles. The one-step fabrication technique involves solvent shifting of aqueous, molecularly dissolved silk using at least a $200 \% v / v$ excess of a water-miscible organic solvent ${ }^{11,20,22}$ in which at least one of the blocks has a low solubility for a resulting supersaturation and spontaneous precipitation..$^{32,33}$

Silk nanoformulations have many advantages, but their manufacture by nanoprecipitation and the understanding of the silk self-assembly mechanism in semi-batch and continuous formats are still progressing. These knowledge gaps are especially pertinent to semi-batch methodology, which can suffer special-cause variation due to differences in experimental scale and even in precipitation vessel design. ${ }^{34,35}$ The scale-up of manufacturing processes required for in vivo clinical trials can cause changes in the production methodology, resulting in aberrant physicochemical characteristics of the nanoparticles ${ }^{35}$ and potentially inaccurate therapeutic and safety profiling. ${ }^{35}$ Consequently, identifying the process parameters which impact silk nanoprecipitation is important. Knowledge of these process parameters can aid in correcting drifts in the physicochemical properties of silk nanoformulations that occur during the transition from lab- to pilot-scale.

Nanoprecipitation occurs due to the interdiffusion of the anti-solvent and water molecules; therefore, it is highly dependent on the mixing conditions, as well as on the composition of the aqueous, solute and anti-solvent components used in the mixture. ${ }^{32,33}$ This raises concerns regarding the commonly accepted lab-scale process of manual drop-by-drop silk feeding to an organic anti-solvent in semi-batch format (e.g. ${ }^{11,36}$ ), as this method results in a time-dependent change in the mixture composition. For this reason, in the last decade, microfluidics has emerged 
as an alternative to dropwise fabrication, as it can provide a continuous route that subjects all solute molecules to the same conditions, thereby allowing increased control over the mixture composition. Microfluidics have been used most extensively for silk water-in-oil emulsions, with narrow polydispersity achieved by flow-focusing droplet microfluidics $(145-200 \mu \mathrm{m})^{37}$ and single T-junction droplet nanofluidics $(51-1500 \mathrm{~nm}){ }^{28}$ However, the micro-size of the emulsions obtained using microchannels ${ }^{37-39}$ limits the application of the resulting particles. The low flow rates required for the generation of nano-sized emulsions can also limit the throughput speed of production on scale-up.

One solution has been the use of the staggered herringbone micromixer, which can provide highthroughput microfluidic-assisted manufacture of nanoparticles $(110-310 \mathrm{~nm})$ using silk nanoprecipitation in acetone and isopropanol anti-solvents ${ }^{12}$. High mixing efficiency occurs in the microchannel as the fluid interface is stretched and folded by asymmetric bas-relief herringbone structures that cause chaotic advection by creating continually switching transverse vortices. ${ }^{40}$ The staggered herringbone micromixer therefore decreases the mixing time compared to laminar flow regimes and semi-batch macromixing. ${ }^{40}$ Use of this micromixer has demonstrated a dependence of the physicochemical properties of the nanoparticles on the solvent properties, the total flow rate and the flow rate ratio of the aqueous and organic phases. ${ }^{12}$

An optimal nanoparticle batch, with a size of $110 \mathrm{~nm}$, a polydispersity of 0.14 , a zeta potential of $-29.8 \mathrm{mV}$, a spherical morphology and aqueous stability, has been obtained in a herringbone micromixer using a flow ratio of 5:1 isopropanol to silk and a total flow rate of $1 \mathrm{~mL} \mathrm{~min}^{-1}{ }^{12}$ These conditions were used in further work to analyse the impact of the precursor molecular weight on nanoparticle properties by varying the sericin degumming time of silk cocoons. ${ }^{13}$ In both manual semi-batch and continuous formats, increasing the degumming time resulted in a greater molecular 
weight polydispersity of the silk stocks and reduced the nanoparticle size, polydispersity and zeta potential. ${ }^{13}$ By comparison, semi-batch production resulted in a significant decrease in surface charge, ${ }^{13}$ indicating that changes in the mixing time and the supersaturation between the two formats impacted the process of silk-silk association.

The aim of the present study was to control the particle characteristics at the lab scale by assessing the parameters that influence silk fibroin nanoparticle assembly under semi-batch and microfluidic mixing regimes. We designed an open and closed system for the semi-batch format and investigated the impact of mixing time and shear by varying several process parameters, including scale, feed addition rate, initial addition height and stirring rate, on the physicochemical properties of the resulting silk nanoparticles. The low and high shear mixing processes in the optimised semi-batch system were then compared with the high shear mixing process in the staggered herringbone micromixer across a range of silk precursor concentrations in order to rationalise the effect of each production route.

\section{EXPERIMENTAL SECTION}

\subsection{Materials}

All studies were carried out at $18-22{ }^{\circ} \mathrm{C}$, unless otherwise stated. Reagents and solvents were acquired from Acros Organics ${ }^{\mathrm{TM}}$ or Sigma Aldrich at $>98 \%$ purity and used without additional purification. Runs for nanoparticle production in continuous format were defined as complete nanoparticle collection using one silk precursor solution $(1 \mathrm{~mL})$. Nanoparticle batches were obtained in continuous format and in semi-batch format using $0.5 \% \mathrm{w} / \mathrm{v}$ aqueous silk by mixing the product suspensions from three runs that used the same silk precursor solution prior to 
ultracentrifugation. Each independent experiment was repeated in triplicate using three different silk precursor stock solutions.

\subsection{Regeneration of $\boldsymbol{B}$. mori silk}

Silk fibroin from $B$. mori cocoons was regenerated using the $1 \mathrm{~h}$ sodium carbonate and $4 \mathrm{~h}$ lithium bromide method detailed previously, ${ }^{41}$ and described in video format elsewhere. ${ }^{11}$

\subsection{General drop-by-drop manufacture of silk nanoparticles in semi-batch format}

Silk nanoparticles were manufactured by the addition of a $3 \% \mathrm{w} / \mathrm{v}$ silk solution to isopropanol in a short-neck round-bottom flask (to give a final 5:1 $v / v$ ratio of isopropanol: silk). The silk feed

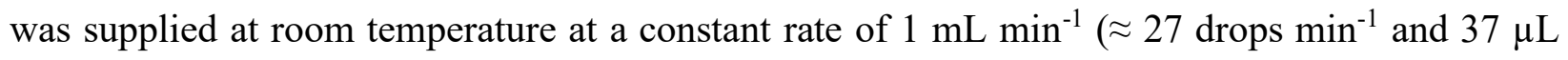
$\min ^{-1}$ ) using a syringe pump (Harvard Apparatus 22, Holliston, MA, USA) held at an inclination of $0-0.1^{\circ}$ and equipped with a BD PLASTIPACK ${ }^{\mathrm{TM}}$ syringe and blunt needle $(0.33 \times 6.35 \mathrm{~mm})$

(Fig. 1a). The flask was stoppered, and the suspension was incubated at room temperature for the designated time before dilution with ultrapure $\mathrm{H}_{2} \mathrm{O}$ in a polypropylene ultracentrifugation tube and centrifugation at $48,400 \times g$ for $2 \mathrm{~h}$ at $4{ }^{\circ} \mathrm{C}\left(\right.$ Beckmann Coulter Avanti ${ }^{\circledR} \mathrm{J}-\mathrm{E}$ equipped with JA-20 rotor). The supernatant was removed, the pellet was resuspended in ultrapure $\mathrm{H}_{2} \mathrm{O}(20 \mathrm{~mL})$ and the suspension was sonicated twice for 30 seconds at 30\% amplitude with a Sonoplus HD 2070 sonicator (ultrasonic homogeniser, Bandelin, Berlin, Germany). Ultrapure $\mathrm{H}_{2} \mathrm{O}(23 \mathrm{~mL})$ was added to the suspension, and the centrifugation, washing and resuspension steps were repeated for a total of three times. The final pellet was suspended in ultrapure $\mathrm{H}_{2} \mathrm{O}(2-3 \mathrm{~mL})$ and stored at $4{ }^{\circ} \mathrm{C}$ until use. Each experiment was repeated in triplicate using three different silk precursor stocks. 
A. Silk pre-processing

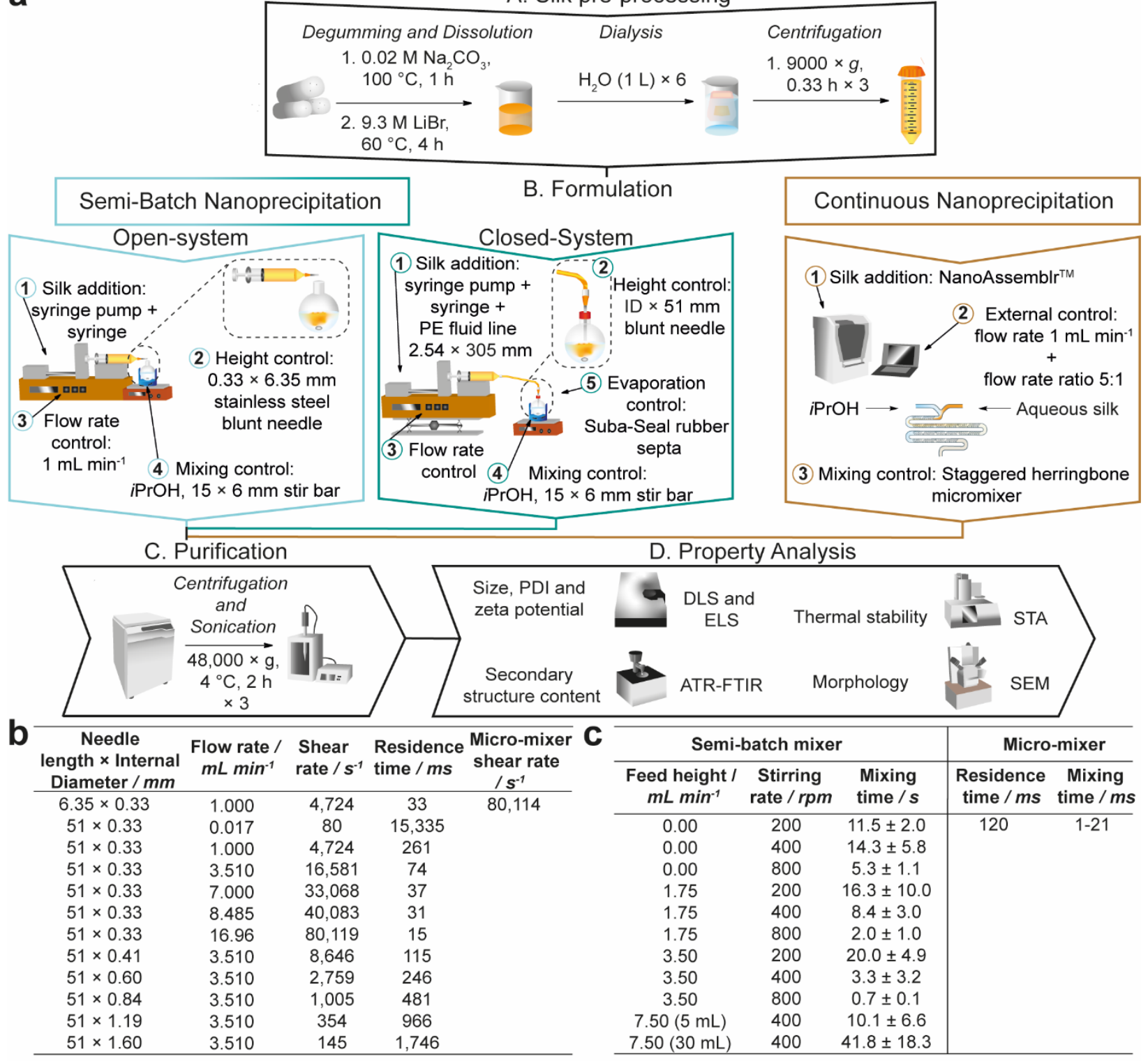

Figure 1. The nanoprecipitation workflow for the preparation and purification of silk nanoparticles via desolvation in isopropanol. (a) In semi-batch format, the four formulation processing steps are: 1) Loading of a bubble-free aqueous silk solution into a syringe equipped with blunt needle. 2) The silk feed position. 3) The flow rate control of silk solution. 4) Control of mixing time via the stirring rate during addition. In continuous format, the three formulation processing steps are: 1) Loading of bubble-free aqueous silk and isopropanol into syringes and the NanoAssemblr ${ }^{\mathrm{TM}}$ microfluidic chip. 2) The flow rate control of silk solution and the flow rate ratio 
control of isopropanol: silk. 3) Control of mixing time via the micromixer chip design. Estimated flow characteristics of the (b) needles used in the open and closed semi-batch system and the micro-mixer, (c) mixing characteristics of the semi-batch reactors and the micromixer. The average wall shear rate under laminar and Newtonian flow is reported. The micromixer geometry was simplified to a rectangular channel by ignoring the groove depth. Residence times in the fluid line and needles were estimated using the linear velocity while the volumetric flow rate was used for the micromixer.

Calculations for needle residence time and shear rate were based on the literature value for dynamic viscosity ( $27 \mathrm{mPas}$ ) of regenerated $3 \%$ aqueous silk ${ }^{42}$ and the calculated density $(1.02 \mathrm{~g}$

$\mathrm{mL}^{-1}$ ) for the $3 \% \mathrm{w} / \mathrm{v}$ aqueous silk solution, with an assumed Newtonian flow (Fig. 1b). ${ }^{42}$ The Reynolds number was estimated as 2 , based on the internal diameter of the needle ${ }^{43}(330 \mu \mathrm{m})$ and indicated laminar flow. An upper limit of the residence time was estimated using the linear velocity $\left(1.94 \mathrm{~mm} \mathrm{~s}^{-1}\right)$ and the needle length. ${ }^{34}$ The maximum shear rate was taken as the wall shear rate and, for simplicity, the shear rate calculations used the geometry of a straight cylinder. Calculations for the 3,10 and $50 \mathrm{~mL}$ syringes used in the study were performed similarly, using the internal diameters stated by the manufacturer (Table S1).

\subsubsection{Ease of scale-up of drop-by-drop silk nanoparticle manufacture}

Silk nanoparticle manufacture was conducted in $5 \mathrm{~mL}$ flasks at the $1.8 \mathrm{~mL}$ scale, in $10 \mathrm{~mL}$ flasks at the $6 \mathrm{~mL}$ scale, in $50 \mathrm{~mL}$ flasks at the 21.9, 36 and $42 \mathrm{~mL}$ scales, and in $150 \mathrm{~mL}$ flasks at the $138 \mathrm{~mL}$ scale. Silk was added from a height of $7.5 \mathrm{~cm}$ from the bottom of the isopropanol meniscus. The mother liquor suspension was incubated for no longer than $2 \mathrm{~h}$ before purification.

\subsubsection{The effects of flow rate and initial addition height in closed, semi-batch format}


Silk nanoparticles were manufactured in $10 \mathrm{~mL}$ round-bottom flasks using a syringe pump (Harvard Apparatus 22, Holliston, MA, USA) equipped with a BD PLASTIPACK ${ }^{\mathrm{TM}}$ syringe, polyethylene Luer lock fluid line $(2.54 \times 305 \mathrm{~mm})$ and blunt needle $(0.33 \times 51 \mathrm{~mm})$. At room temperature, a 3\% w/v silk solution $(1 \mathrm{~mL})$ was added to isopropanol $(5 \mathrm{~mL})$ at flow rates of 0.017 , $1.000,3.510$ or $7.00 \mathrm{~mL} \mathrm{~min}^{-1}$, while varying the initial addition height at $0.7,2.1$ or $3.5 \mathrm{~cm}$ from the isopropanol surface. The Reynolds numbers at $0.017,1.000,3.510$ and $7.000 \mathrm{~mL} \mathrm{~min}^{-1}$ (calculated as $5.4 \times 10^{-3}, 0.32,1.1$ and 2.2 for the fluid line, and as $0.04,2.5,8.5$ and 17 for the needle) indicated laminar flow (Table S2, Fig. 1b). The mother liquor suspension was incubated for no longer than 30 min before purification.

\subsubsection{The effect of needle diameter in the closed, semi-batch format}

Silk solution was added at a rate of $3.51 \mathrm{~mL} \mathrm{~min}^{-1}$ to isopropanol from an initial addition height of $3.5 \mathrm{~cm}$ from the isopropanol surface. The needle diameter was varied between $0.33,0.41,0.60$, $0.84,1.19$ and $1.60 \mathrm{~mm}$. The Reynolds numbers for the needle diameters of $0.41,0.60,0.84,1.19$ and 1.60 were calculated as $6.9,4.7,3.4,2.4$ and 1.8 , respectively. The mother liquor suspension was incubated for no longer than 30 min before purification.

\subsubsection{The effect of stirring rate and feed addition height in the closed, semi-batch format}

Silk nanoparticles were manufactured using a blunt needle $(0.33 \times 51 \mathrm{~mm})$. The silk solution flow rate was $1.00 \mathrm{~mL} \mathrm{~min}^{-1}$, the initial addition height was $0.0,1.75$ or $3.5 \mathrm{~cm}$ from the isopropanol surface, and the stirring rate, provided by an egg-shaped stir bar $(15 \times 6 \mathrm{~mm})$, was $0,200,400$ or $800 \mathrm{rpm}$. The Reynolds number of the stirred vessel, estimated using a cylindrical geometry as $\approx$ 1300, 2500 and 5030 at stirring rates of 200, 400 and $800 \mathrm{rpm}$, respectively (Table S3), indicated turbulent flow within the vessel at 400 and $800 \mathrm{rpm}$.

\subsubsection{The effect of flow rate and concentration in the closed, semi-batch format}


Silk nanoparticles were manufactured using a blunt needle $(0.33 \times 51 \mathrm{~mm})$. Silk solution was added to isopropanol at rates of $0.017,1.000,8.485$ or $16.96 \mathrm{~mL} \mathrm{~min}^{-1}$ from an addition height of $1.75 \mathrm{~cm}$ and stirred at $400 \mathrm{rpm}$ with the egg-shaped stir bar. The silk concentration was $0.5,2$ or $3 \% \mathrm{w} / \mathrm{v}$. The Reynolds numbers at 8.485 and $16.96 \mathrm{~mL} \mathrm{~min}^{-1}$ (calculated as 2.7 and 5.4 for the fluid line and 21 and 41 for the needle) indicated laminar flow. The mother liquor suspension was incubated for no longer than 30 min before purification.

\subsection{Dual indicator system for mixing time in the semi-batch format}

The rotational speed investigated at the $5 \mathrm{~mL}$ scale ranged from $200-800 \mathrm{rpm}$ in increments of 200 $\mathrm{rpm}$, and the initial addition height was $0,1.75,3.5$ or $7.5 \mathrm{~cm}$. The rotational speed and the initial addition height at the $30 \mathrm{~mL}$ scale were fixed at $400 \mathrm{rpm}$ and $7.5 \mathrm{~cm}$, respectively. The round bottom flask was submerged in water in a clear acrylic box $(10.3 \times 10.3 \times 5 \mathrm{~cm})$ to reduce surface reflections. An LED panel (RALENO, Seattle, WA, USA) was fixed at the back of the stirring plate to provide constant illumination at $5600 \mathrm{~K}$ colour temperature and at $100 \%$ brightness.

The dual indicator system for mixing time described by Melton et al. ${ }^{44}$ and Weheliye et $a l .{ }^{45,46}$ was used, with some adaptations. Stock solutions of thymol blue $\left(0.095 \mathrm{mg} \mathrm{mL}^{-1}\right)$ and methyl red $\left(0.135 \mathrm{mg} \mathrm{mL}^{-1}\right)$ were prepared in ethanol. The working solution was prepared by mixing and diluting the stock solutions to give $4.3 \mathrm{mg} \mathrm{mL}^{-1}$ thymol blue and methyl red in $70 \% \mathrm{v} / \mathrm{v}$ ethanol. To each 5 or $30 \mathrm{~mL}$ aliquot of the working solution, $0.5 \mathrm{M} \mathrm{HCl}$ was added at $0.5 \mathrm{~mL} / \mathrm{L}$, and the system was equilibrated for at least 10 revolutions. An equivalent amount of $\mathrm{NaOH}(10.5 \mu \mathrm{L}$ of $0.15 \mathrm{M} \mathrm{NaOH}$ for $5 \mathrm{~mL}$ aliquots and $10.5 \mu \mathrm{L}$ of $0.71 \mathrm{M} \mathrm{NaOH}$ for $30 \mathrm{~mL}$ aliquots) was then added to the mixture using a $20 \mu \mathrm{L}$ Eppendorf pipette (attached to a clamp stand for control of the feed location and height). The mixing process was captured on an iPhone SE (Apple, Cupertino, CA, 
USA) reverse camera at a capture speed and resolution of $240 \mathrm{fps}$ and $1080 \mathrm{p}$ using FiLMiC Pro (FiLMiC Inc., Seattle, WA, USA). Each condition was repeated at least four times.

Images were extracted at 240 fps using FFmpeg. ${ }^{47}$ The images were processed using custom MATLAB (Mathworks, Natick, USA) scripts to apply rectangular masks of 18,000 pixels and to calculate the standard deviation of the normalised green channel intensity, as described by Rodriguez et al. ${ }^{48}$ The standard deviation of the fully mixed condition was calculated as the average of the final ten images, and the mixing time ( $\mathrm{t}_{95 \%}$ ) was estimated as the time required to reach $95 \%$ of the standard deviation at the fully mixed condition (Fig. 1c).

\subsection{Microfluidic-assisted manufacture of silk nanoparticles}

Silk nanoparticles were manufactured using the NanoAssemblr ${ }^{\mathrm{TM}}$ benchtop instrument version 1.5 (model number NA-1.5-16; NanoAssemblrTM, Precision Nano-Systems Inc. Vancouver, Canada) equipped with a cyclic olefin copolymer microfluidic cartridge (product codes: 1207 and 1151034 BENCHTOP CARTRIDGE), as described elsewhere. ${ }^{12}$ Using the Y-junction of the two 25 $\mathrm{mm}$ inlet channels, the fluids were mixed in a $27 \mathrm{~mm}$ rectangular mixing channel $(79 \mu \mathrm{m} \times 200$ $\mu \mathrm{m})$ having a series of raised grooves $(31 \mu \mathrm{m} \times 50 \mu \mathrm{m})$ and four switchback turns. ${ }^{49} \mathrm{~A} 1 \mathrm{~mL}$ volume of aqueous silk solution $(0.5-3 \% \mathrm{w} / \mathrm{v})$ and $5 \mathrm{~mL}$ isopropanol were injected into separate chamber inlets, and the nanoparticles formed in the staggered herringbone mixer and were

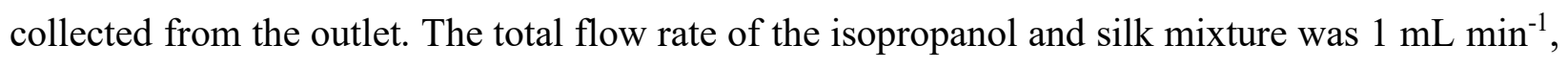
and the flow rate ratio was 5:1.

The cartridge was cleaned between runs using a water wash and a prime. The water wash consisted of a flow ratio of 1:1 ultrapure $\mathrm{H}_{2} \mathrm{O}$ /ultrapure $\mathrm{H}_{2} \mathrm{O}$, a total volume of $2 \mathrm{~mL}$ and a total flow rate of $4 \mathrm{~mL} \mathrm{~min}^{-1}$; the wash was repeated in triplicate. The priming procedure consisted of a flow ratio of 5:1 isopropanol/ultrapure $\mathrm{H}_{2} \mathrm{O}$, a total volume of $6 \mathrm{~mL}$ and a total flow rate of $1 \mathrm{~mL}$ 
$\min ^{-1}$. The mother liquor suspension was incubated for no longer than $2 \mathrm{~h}$ before purification. The optimum formulation parameters were used in a round robin test in the NanoAssemblr ${ }^{\mathrm{TM}}$ to estimate the repeatability and reproducibility upon parallelisation. Results from four participants and microfluidic chips (Fig. S1a-c) were analysed for consistency and precision according to ASTM E $69 .{ }^{50}$

Calculations were based on the literature viscosity value $(3.14 \mathrm{mPas})$ and density $\left(0.837 \mathrm{~g} \mathrm{~mL}^{-}\right.$

${ }^{1}$ ) values for the $5: 1 \mathrm{v} / \mathrm{v}$ isopropanol/water mixture measured at $20{ }^{\circ} \mathrm{C},{ }^{51}$ and Newtonian flow was assumed. ${ }^{42}$ The mixing time was estimated at $1-21 \mathrm{~ms}$, according to the manufacturer's guidelines and based on an analytical model for a similar system published elsewhere, by calculating the Peclet number to achieve a coefficient of variation of $<0.1$ (Fig. 1c). ${ }^{52}$ The Peclet number was estimated as $4.27 \times 10^{11}$ using the hydraulic diameter of the channel ${ }^{43}(142 \mu \mathrm{m})$ and the diffusion coefficient $\left(3.5 \times 10^{-10} \mathrm{~m}^{2} \mathrm{~s}^{-1}\right)$ of the 5:1 isopropanol/water mixture ${ }^{53}$. The Reynolds number (40) indicated laminar flow. An upper limit of the residence time was estimated using the total fluidic volume and flow rate. ${ }^{34}$ The residence time was longer than the mixing time, indicating that complete mixing had occurred in the micromixer. The maximum shear rate was taken as the wall shear rate, with the assumptions that chaotic advection did not significantly affect the wall shear rate and that it created a significantly lower shear within the channel. For simplicity, the shear rate calculations used the geometry of a straight rectangular channel and did not take into account the groove depth. ${ }^{54}$

\subsection{Yield of silk nanoparticles}

The total mass of silk nanoparticles was recorded by transferring $450 \mu \mathrm{L}$ of the suspension to a pre-weighed microcentrifuge tube and recording the total mass, followed by freezing at $-80{ }^{\circ} \mathrm{C}$ for $5 \mathrm{~h}$ and freeze-drying (Christ Epsilon 1-4, Martin Christ Gefriertrocknungsanlagen GmbH, 
Osterode, Germany) for $24 \mathrm{~h}$ at $-10{ }^{\circ} \mathrm{C}$ and 0.14 mbar. The dry mass was then recorded and the yield calculated as detailed previously. ${ }^{41}$ This process was repeated twice, and an average yield was reported. Freeze-dried silk nanoparticles were stored in a vacuum desiccator at $25{ }^{\circ} \mathrm{C}$ until use.

\subsection{Physicochemical characterization of the silk nanoparticles and stability in water}

The silk nanoparticle size (Z-average of the hydrodynamic diameter), polydispersity and zeta potential were measured in triplicate in ultrapure $\mathrm{H}_{2} \mathrm{O}$ at $25^{\circ} \mathrm{C}$ by dynamic light scattering (DLS) (Zetasizer Nano-ZS Malvern Instrument, Worcestershire, UK). Nanoparticle suspensions were prepared for measurement by vortex application for 20 seconds and sonication twice at $30 \%$ amplitude for 30 seconds, unless otherwise stated. Refractive indices of 1.33 and 1.60 were used for $\mathrm{H}_{2} \mathrm{O}$ and protein, respectively.

The silk nanoparticles from all studies were stored at $4^{\circ} \mathrm{C}$ and the particle size and zeta potential of silk nanoparticles generated in the open, semi-batch system and the NanoAssemblr ${ }^{\mathrm{TM}}$ were determined on days 0 to 42 by DLS. The nanoparticles were prepared for measurement by vortexing for 20 seconds at $t>0$ days.

\subsection{Secondary structure measurements of silk nanoparticles}

Positive silk II controls consisted of autoclaved silk films and silk films treated with 70\% v/v ethanol/ultrapure $\mathrm{H}_{2} \mathrm{O}$. Positive silk I controls were air-dried silk films and freeze-dried silk. The air-dried silk films were drop-casted from fluid handling systems following extrusion at a height of $3.5 \mathrm{~cm}$ using varying silk volumes $(0.3-7 \mathrm{~mL})$, silk concentrations $(0.5-3.0 \% \mathrm{w} / \mathrm{v})$, flow rates $\left(0.017-16.96 \mathrm{~mL} \mathrm{~min}^{-1}\right)$ and needle diameters $(0.33-1.60 \mathrm{~mm})$. The secondary structures of silk films, freeze-dried powders and freeze-dried nanoparticles were analysed by Fourier transform infrared spectroscopy (FTIR) on an ATR-equipped TENSOR II FTIR spectrometer (Bruker Optik 
GmbH, Ettlingen, Germany). Each FTIR measurement was recorded in absorption mode over the wavenumber range of 400 to $4000 \mathrm{~cm}^{-1}$ at $4 \mathrm{~cm}^{-1}$ resolution for 128 scans and then corrected for atmospheric absorption using Opus (Bruker Optik GmbH, Ettlingen, Germany). The second derivatives of the background-corrected FTIR absorption spectra were analysed in OriginLab 19b ${ }^{\circledR}$ (Northampton, Massachusetts, USA) by adapting a literature protocol. ${ }^{55}$ Each second derivative was smoothed twice using a seven-point Savitzky-Golay function with a polynomial order of 2. The amide I region was analysed by interpolation of a non-zero linear baseline between $2-3$ of the highest values in the $1600-1700 \mathrm{~cm}^{-1}$ range. Peak positions were identified by applying the second derivative, and peaks were fitted in the amide I region using non-linear least squares with a series of Gaussian curves (Fig. S2). The position, width and height of each peak were allowed to vary, while the peak area could take any value $\leq 0$. Deconvoluted spectra were then area-normalised, and the relative area of each band was used to calculate the secondary structure content according to literature band assignments. ${ }^{38,56}$

The correlation coefficients $(\mathrm{R})$ of silk films, freeze-dried silk and nanoparticles were calculated by adaptation of a literature protocol ${ }^{57}$ using the air-dried silk film of an aqueous silk precursor batch as a reference. The second derivative of the FTIR absorption spectra was calculated and smoothed twice with a five-point Savitzky-Golay function and a polynomial order of 2 . The processed silk sample was then compared with the reference over the spectral range 1600-1700 $\mathrm{cm}^{-1}$ using equation 1 .

$\mathrm{R}=\frac{\sum \mathrm{x}_{\mathrm{i}} \mathrm{y}_{\mathrm{i}}}{\sqrt{\sum \mathrm{x}_{\mathrm{i}}^{2} \sum \mathrm{y}_{\mathrm{i}}^{2}}}(1)$

where $x_{i}$ and $y_{i}$ are the derivative values of the air-dried silk film and processed silk sample at the frequency i.

\subsection{Thermal analysis of silk nanoparticles}


Freeze-dried silk nanoparticle samples (1.06-4.89 mg) in aluminium pans were subjected to differential scanning calorimetry (DSC) and thermogravimetric analysis (TGA) from $20-350{ }^{\circ} \mathrm{C}$ at a scanning rate of $10{ }^{\circ} \mathrm{C} \mathrm{min}^{-1}$ and a nitrogen flow of $50 \mathrm{~mL} \mathrm{~min}^{-1}$ (STA Jupiter 449, Netzsch, Gerätebau GmbH, Germany). Thermograms were analysed using OriginLab 19b ${ }^{\circledR}$ (Northampton, Massachusetts, USA). According to previous descriptions, ${ }^{58}$ the desorption enthalpy was normalised to the corrected mass.

\subsection{Scanning electron microscopy of silk nanoparticles}

A $1 \mathrm{mg} \mathrm{mL} \mathrm{m}^{-1}$ silk nanoparticle suspension $(10-20 \mu \mathrm{L})$ was pipetted onto a silicon wafer and lyophilised at $-10{ }^{\circ} \mathrm{C}$ and 0.14 mbar for $24 \mathrm{~h}$. The wafer was sputter-coated with gold $(15 \mathrm{~nm})$ using a low vacuum sputter coater (Agar Scientific Ltd, Essex, UK, and ACE200, Leica Microsystems, Wetzlar, Germany) and imaged with an FE-SEM SU6600 instrument (Hitachi High Technologies, Krefeld, Germany) at $5 \mathrm{kV}$ and $40 \mathrm{k}$ magnification. The images were processed using ImageJ v1.52n (National Institutes of Health, Bethesda, MD, U.S.A), Adobe Lightroom and Abode Illustrator (Adobe, San Jose, CA, USA).

\subsection{Statistical analyses}

Data were analysed using Microsoft ${ }^{\circledR}$ Excel ${ }^{\circledR} 2019$ (Microsoft Office 365 ProPlus Software, Redmond, WA, U.S.A), Minitab ${ }^{\circledR}\left(\right.$ Minitab ${ }^{\circledR}$ Statistical Software, State College, PA, USA) and GraphPad Prism 8.2.1 (GraphPad Software, La Jolla, CA, U.S.A.). Normality of the data distributions was assumed throughout. The equivalence of variance for sample pairs and multiple groups was identified with the F-test and Bartlett's test. Sample pairs were analysed using the independent $t$-test. Multiple groups across one independent variable were evaluated by one-way analysis of variance (ANOVA), followed by Tukey's pairwise multiple comparison post-hoc test, or by the Brown-Forsythe and Welch ANOVA tests, followed by the Dunnett T3 pairwise multiple 
comparison post-hoc test. Two-way ANOVA was used to compare multiple groups across two independent variables, followed by Tukey's pairwise multiple comparison post-hoc test when a significant interaction was shown and by Tukey's main and simple effect multiple comparison post-hoc tests when no interaction was shown. Silk nanoparticle stability was evaluated by ANOVA, followed by Dunnett's or the Dunnett T3 post hoc test, to compare between $t=0$ day control and $t>0$ day samples. Statistical significance, identified using post-hoc tests, was as follows: $* \mathrm{p}<0.05, * * \mathrm{p}<0.01, * * * \mathrm{p}<0.001, * * * * \mathrm{p}<0.0001$. All data are displayed as the mean \pm standard deviation, with the number of experimental repeats $(n)$ shown in each figure legend.

\subsection{RESULTS}

\subsection{Silk nanoparticle characterisation}

\subsubsection{The effect of scale and manufacturing process}

In the semi-batch open system, the physicochemical properties of silk nanoparticles were determined by the scale of nanoprecipitation in the presence or absence of stirring. In the absence of stirring, an increase in scale from 1.8 to $21.9 \mathrm{~mL}$ significantly decreased the silk nanoparticle size from $149 \mathrm{~nm}$ to $116 \mathrm{~nm}$ (Fig. 2a). No further reduction in size occurred following further increases in the scale from 21.9 to $138 \mathrm{~mL}$. The zeta potential significantly decreased from -31 to $-39 \mathrm{mV}$ as the scale increased from 6 to $138 \mathrm{~mL}$ (Fig. 2a). However, the polydispersity index, yield and spherical morphology were not affected by the scale (Fig. 2a-c). Increasing the stirring rate from 0 to $400 \mathrm{rpm}$ at the $6 \mathrm{~mL}$ scale significantly reduced the nanoparticle size from $134 \mathrm{~nm}$ to $114 \mathrm{~nm}$ and the yield from $23 \%$ to $9 \%$ (Fig. 2d). In contrast to the $6 \mathrm{~mL}$ scale result, stirring had no similar impact when the scale was increased to $36 \mathrm{~mL}$ (Fig. 2d). 


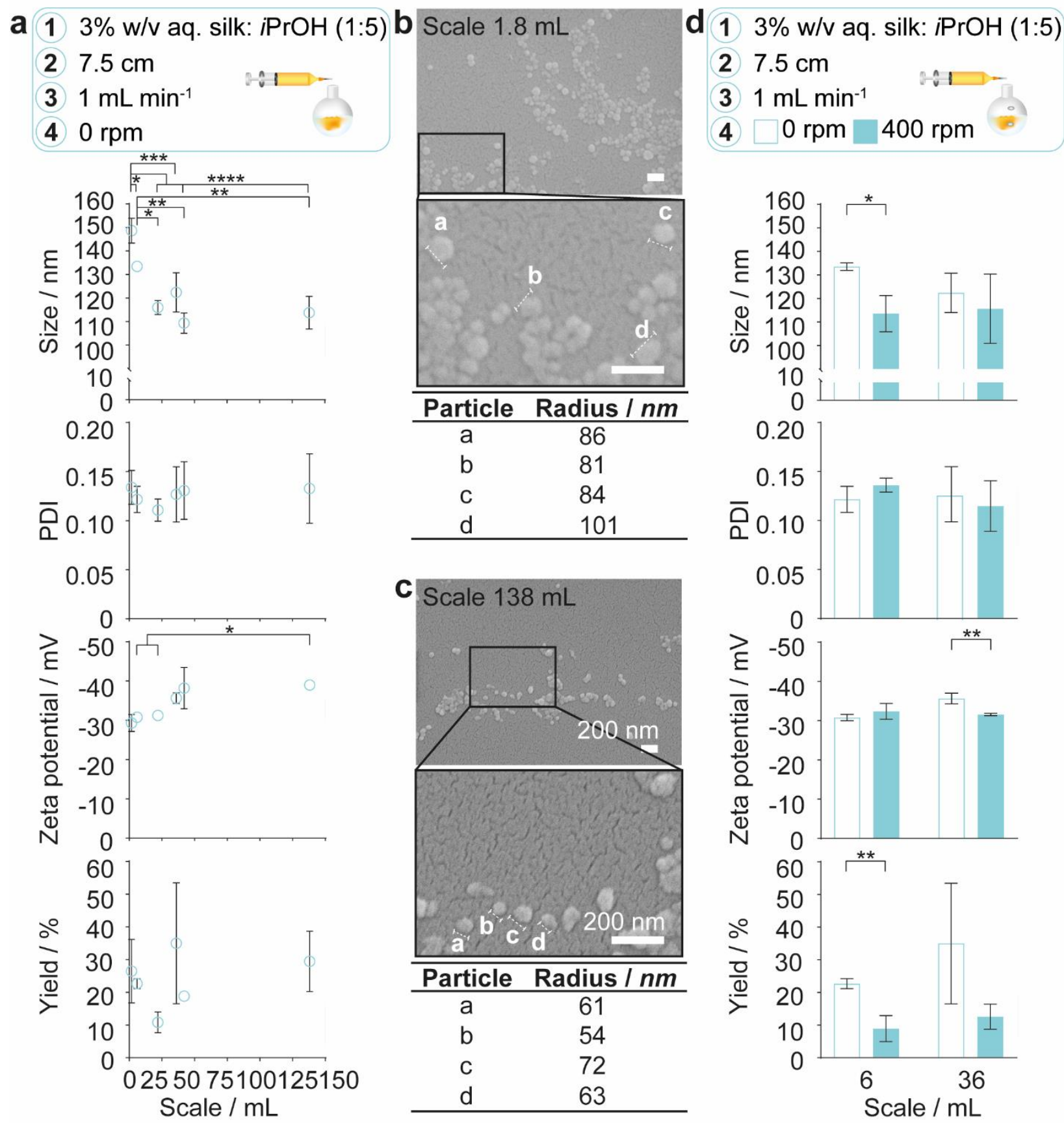

Figure 2. The impact of semi-batch manufacturing scale on nanoparticle physicochemical properties and yield. (a) As the scale of silk desolvation in semi-batch format increased without stirring, nanoparticle size decreased, and zeta potential increased significantly. Multiple groups were evaluated by one-way ANOVA, followed by Tukey's multiple comparison post-hoc test. Scanning electron microscopy showed that nanoparticles manufactured at (b) 1.8 and (c) $138 \mathrm{~mL}$ 
were spherical. (d) As stirring rate increased at $6 \mathrm{~mL}$ scale, nanoparticle size and yield decreased, while zeta potential increased with stirring rate at $36 \mathrm{~mL}$ scale. Multiple groups were evaluated by a Student's t-test. Data obtained from semi-batch manufacture at $6 \mathrm{~mL}$ scale and at $36 \mathrm{~mL}$ scale at the stirring rate of $400 \mathrm{rpm}$ have been published elsewhere. ${ }^{41}$ Error bars are hidden in the plot symbols when not visible, $\pm \mathrm{SD}, n=3$. Asterisks denote statistical significance determined using post-hoc tests as follows: $* \mathrm{p}<0.05,{ }^{* *} \mathrm{p}<0.01,{ }^{* * *} \mathrm{p}<0.001,{ }^{* * * *} \mathrm{p}<0.0001$. Scale bars $200 \mathrm{~nm}$.

Unlike the semi-batch format, continuous nanoprecipitation in the staggered herringbone micromixer was a scale-independent process. For this reason, we used the optimum formulation parameters in a round robin test at the $6 \mathrm{~mL}$ scale to estimate the operating boundaries upon parallelisation. The between-participant and within-participant consistencies were characterised by the $\mathrm{h}$ and $\mathrm{k}$ statistics, respectively (Fig. S1). No outliers were observed, as the data did not exceed the critical $\mathrm{h}$ and $\mathrm{k}$ values. The precision statistics of nanoparticle properties indicated that, for one microfluidic chip and operator, the absolute differences in nanoparticle size, polydispersity index and zeta potential of $13 \mathrm{~nm}, 0.06$ and $5 \mathrm{mV}$, respectively, between two nanoprecipitation results would be expected to occur with a $95 \%$ probability (Table S4). For multiple microfluidic chips, the analogous differences were $17 \mathrm{~nm}, 0.09$ and $11 \mathrm{mV}$, respectively.

\subsubsection{The effect of flow rate, addition height and stirring rate in closed, semi-batch format}

A closed, semi-batch system was then designed to reduce solvent evaporation during the addition of the silk feed. In the absence of stirring, increasing the flow rate of the silk solution from 0.017 $\mathrm{mL} \min ^{-1}$ (mixing-induced nucleation) to $7.000 \mathrm{~mL} \mathrm{~min}^{-1}$ (shear-induced nucleation), while also increasing the silk feed addition height, resulted in large, polydisperse nanoparticles regardless of factor levels (Fig. S3a). Although the nanoparticles remained large and polydisperse, scanning 
electron microscopy indicated that moving from shear-induced to mixing-induced nucleation by increasing the needle diameter reduced the extent of self-assembly (Fig. S3c-d)

As manufacture in the closed, semi-batch system without stirring resulted in large nanoparticles with a wide size distribution, the effect of varying the time and shear stress of mixing was then determined at a $1.000 \mathrm{~mL} \mathrm{~min}^{-1}$ flow rate (shear-induced nucleation). The mixing time was reduced by increasing the stirring rate from 0 to $800 \mathrm{rpm}$ and increasing the feed height from $0.0 \mathrm{~cm}$ ( $v_{\text {droplet }}$ $\left.\approx 0.0019 \mathrm{~ms}^{-1}\right)$ to $1.75 \mathrm{~cm}\left(v_{\text {droplet }} \approx 0.59 \mathrm{~ms}^{-1}\right)$ and $3.5 \mathrm{~cm}\left(v_{\text {droplet }} \approx 0.83 \mathrm{~ms}^{-1}\right)$ (Fig. 1c and Fig. 3). Increasing the levels of both these factors significantly reduced the nanoparticle size and polydispersity index (Fig. 3a). At $0 \mathrm{rpm}$, an increase in the feed height from 0.0 to $3.5 \mathrm{~cm}$ significantly decreased the nanoparticle size (from 232 to $151 \mathrm{~nm}$ ) and the polydispersity index (from 0.24 to 0.12 ) (Fig. 3a). Equally, an increase in the stirring rate from 0 to $800 \mathrm{rpm}$ significantly decreased the nanoparticle size to $116 \mathrm{~nm}$ at $0.0 \mathrm{~cm}$ feed height and the polydispersity index from 0.25 to 0.14 at $1.75 \mathrm{~cm}$ feed height. Scanning electron microscopy confirmed the reduction in assembly size and the increase in nanoparticle curvature as mixing time decreased (Fig. 3b). 
a

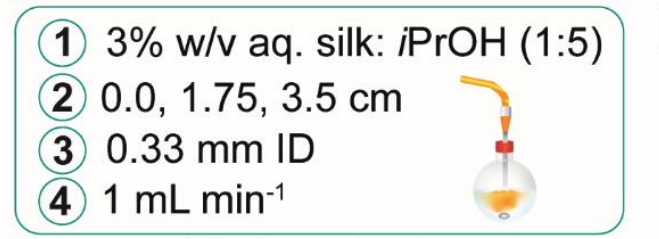

$\square 0 \quad \begin{array}{lr}\text { Stirring rate } / \mathrm{rpm} \\ \square 0\end{array}$

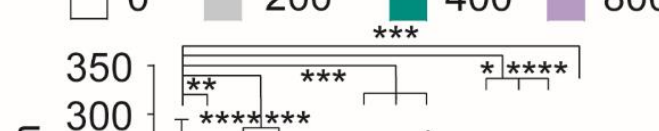

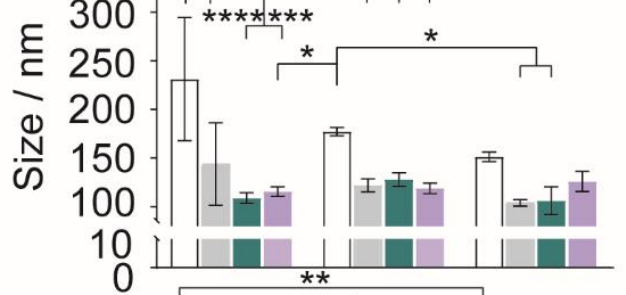
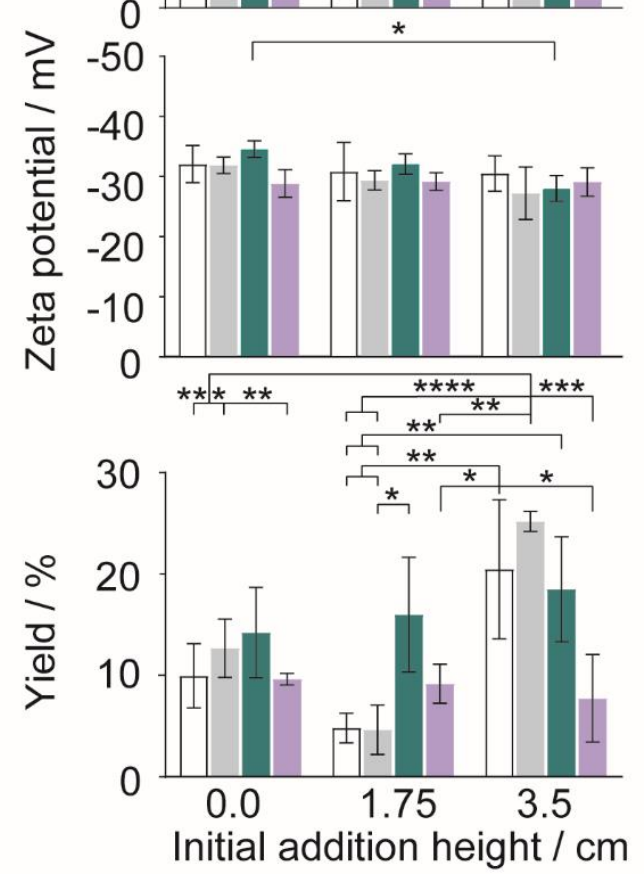
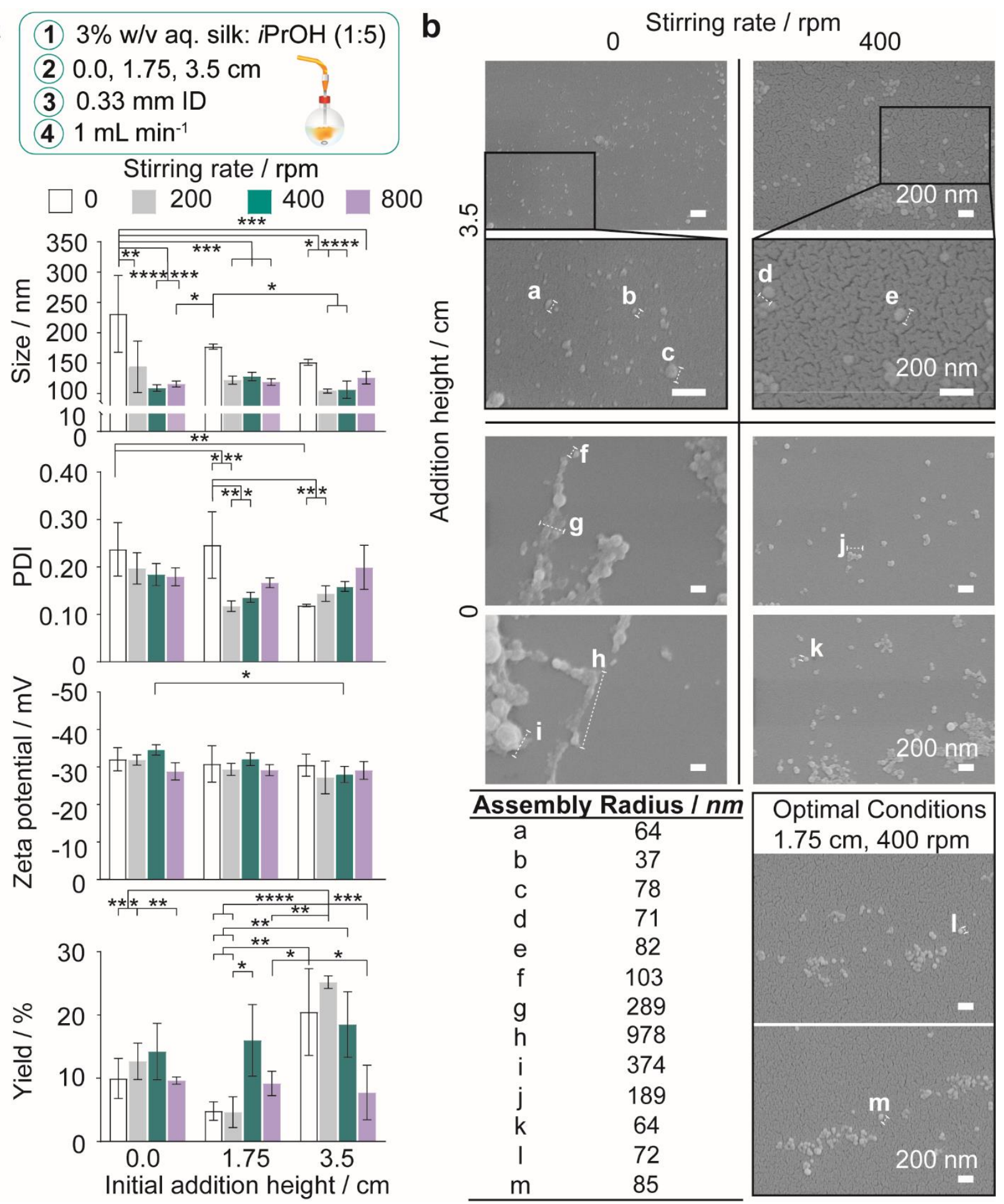

Figure 3. Impact of feed height and stirring rate on nanoprecipitation of $3 \% \mathrm{w} / \mathrm{v}$ aqueous

silk in the semi-batch closed-system at $1 \mathrm{~mL} \mathrm{~min}$ min $^{-1}$ flow rate. (a) Hydrodynamic diameter, polydispersity index (PDI), zeta potential and yield of silk nanoparticles. Two-way ANOVA was 
used to compare multiple groups across feed height and stirring rate, followed by Tukey's pairwise multiple comparison post-hoc test for size, polydispersity index and yield and Tukey's simple effect multiple comparison post-hoc test for zeta potential. (b) Scanning electron microscopy showed that lower curvature morphologies were obtained as stirring rate and addition height decreased, due to an increasing degree of secondary self-assembly. Error bars are hidden in the bars and plot symbols when not visible, $\pm \mathrm{SD}, n=3$. Asterisks denote statistical significance determined using post-hoc tests as follows: ${ }^{*} \mathrm{p}<0.05, * * \mathrm{p}<0.01, * * * \mathrm{p}<0.001, * * * * \mathrm{p}<0.0001$. Scale bars $200 \mathrm{~nm}$.

Generally, the nanoparticle size and size distribution were optimal at 1.75 and $3.5 \mathrm{~cm}$ feed heights at stirring rates of 200 and $400 \mathrm{rpm}$ (104-128 nm; 0.12-0.16) (Fig. 3a). As feed height increased, silk nanoparticles were also produced with a higher zeta potential at $400 \mathrm{rpm}$, and in greater yield at low stirring rates, where the yield reached a maximum of $25 \%$ at $3.5 \mathrm{~cm}$ and 200 rpm. Further reductions in the mixing time at $3.5 \mathrm{~cm}$ (Fig. 1c) obtained by increasing the stirring rate to $800 \mathrm{rpm}$ caused a significant drop in nanoparticle yield to $8 \%$. Overall, the nanoparticle size, polydispersity index and zeta potential were optimised at $1.75 \mathrm{~cm}$ feed height and $400 \mathrm{rpm}$ stirring rate and a sufficient yield was returned (Fig. 3a). These factor levels were set for further investigations of the effect of flow rate and silk concentration.

\subsubsection{The effect of flow rate and silk concentration in closed, semi-batch and microfluidic}

\section{formats}

Under conditions of mixing-induced nanoprecipitation in semi-batch format at a $0.017 \mathrm{~mL} \mathrm{~min}^{-1}$ flow rate, increasing the silk concentration from 0.5 to 2 and $3 \%$ significantly decreased the nanoparticle size (from 271 to 89 and $75 \mathrm{~nm}$ ) and polydispersity index (from 0.47 to 0.23 and 0.13 ) 
(Fig. 4a). Scanning electron microscopy revealed an increase in particle sphericity with increasing concentration, from the nanofibers and gel-like aggregates present at $0.5 \%$ to spherical particles with a high polydispersity at $2 \%$ and a narrower size distribution at $3 \%$ (Fig. 4 b).

a

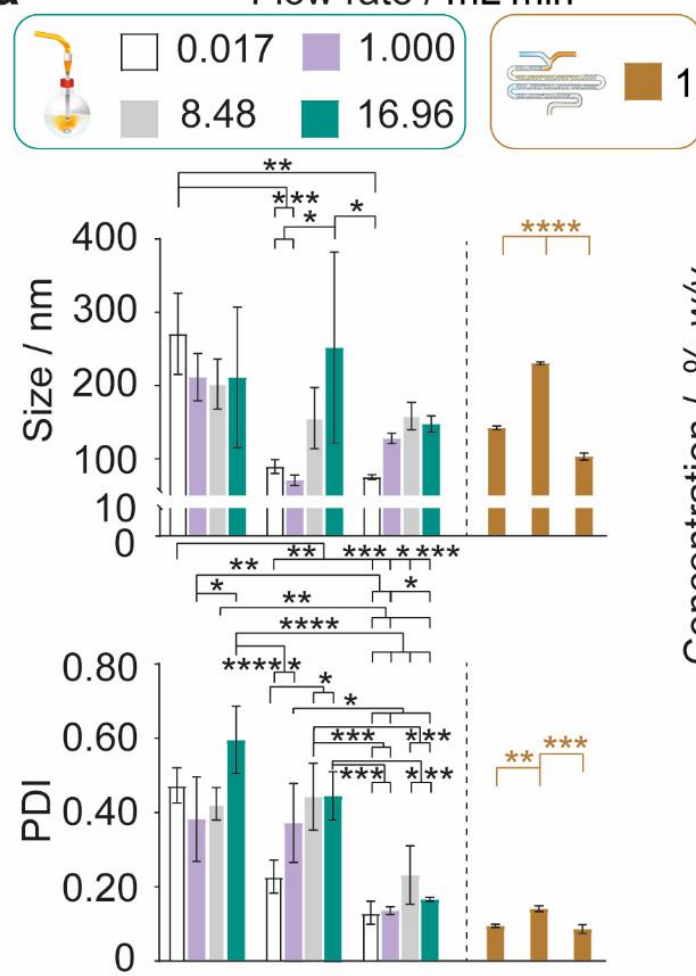

b Semi-batch

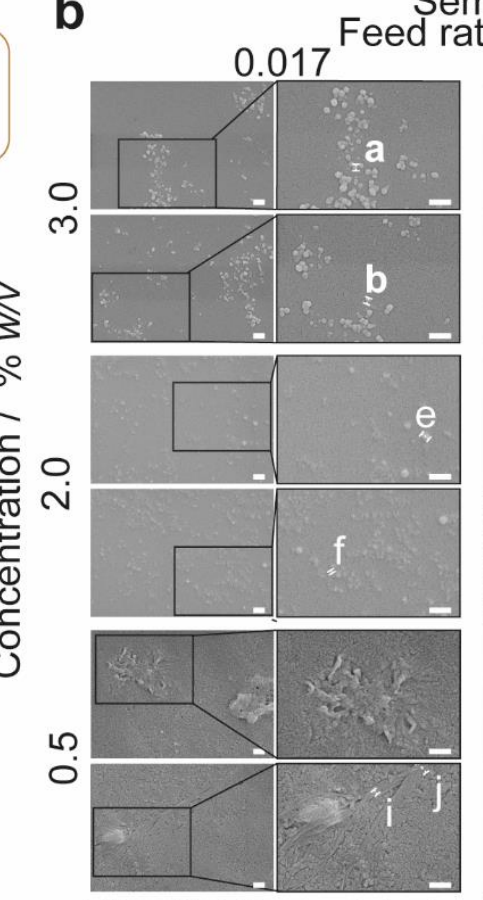

Microfluidic-assisted

Total flow rate $1 \mathrm{~mL} \mathrm{\textrm {min } ^ { - 1 }}$

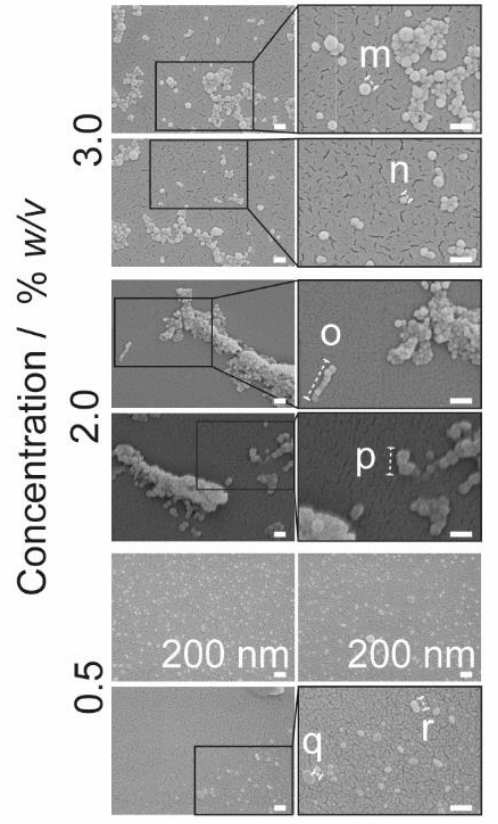

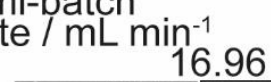

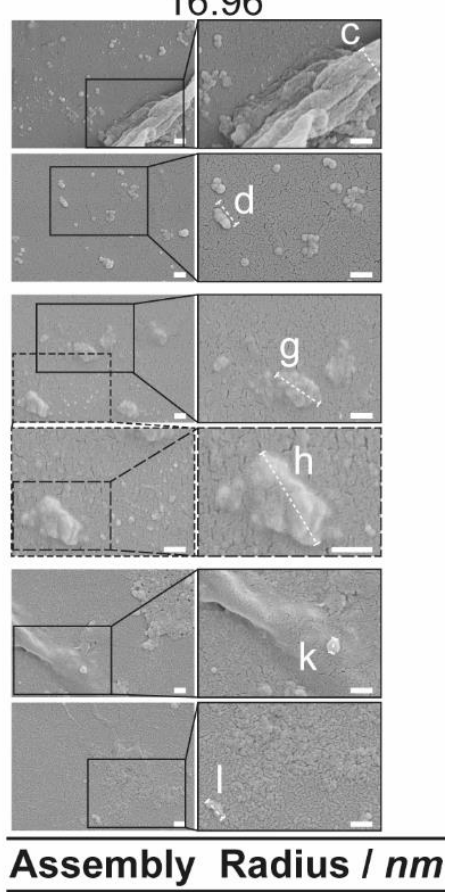

53

54

343

236

87

37

463

504

30

75

130

193

75

122

373

143

65

125

Figure 4. Impact of silk concentration on nanoparticle manufacture using closed, semi-batch 
and microfluidic formats. (a) Hydrodynamic diameter, polydispersity index (PDI), zeta potential and yield. For semi-batch format, two-way ANOVA was used to compare multiple groups across concentration and flow rate, followed by Tukey's pairwise multiple comparison post-hoc test for size, polydispersity index and yield and Tukey's simple effect multiple comparison post-hoc test for zeta potential. In microfluidic format, multiple groups were evaluated by one-way ANOVA, followed by Tukey's multiple comparison post-hoc test. (b) Scanning electron microscopy showed that curvature morphology decreased with concentration in semi-batch and microfluidic-assisted formats and varied inversely with flow rate in semi-batch format. Error bars are hidden in the bars when not visible, $\pm \mathrm{SD}, n=3$. Asterisks denote statistical significance determined using post-hoc tests as follows: ${ }^{*} \mathrm{p}<0.05,{ }^{* *} \mathrm{p}<0.01,{ }^{* * *} \mathrm{p}<0.001,{ }^{* * * *} \mathrm{p}<0.0001$. Scale bars $200 \mathrm{~nm}$.

Moving from low to high shear by increasing the flow rate from 0.017 to $16.96 \mathrm{~mL} \mathrm{~min}^{-1}$ caused a significant increase in the nanoparticle size from 89 to $252 \mathrm{~nm}$ with $2 \%$ silk. The size distribution also significantly increased with flow rate at silk concentrations of 0.5 and $2 \%$. Scanning electron microscopy confirmed that these results were due to a greater degree of shear-induced selfassembly with increased flow rate across all concentrations (Fig. 4b). For example, at 3\%, tertiary units were present as nanofibers, while at $0.5 \%$ and $2 \%$, the formation of gel-like aggregates also occurred. As for mixing-induced nanoprecipitation, the polydispersity index decreased significantly with increased concentration across all high shear flow rates. The yield increased significantly with concentration at $1 \mathrm{~mL} \mathrm{~min}^{-1}$ and reached a maximum at $3 \%$ (from 1 to $16 \%$ ).

Similarly, in the high-shear microfluidic format, increasing the silk concentration from $0.5 \%$ to $2 \%$ resulted in a significant increase in particle size, from $142 \mathrm{~nm}$ to $231 \mathrm{~nm}$; polydispersity index, from 0.10 to 0.19 ; and zeta potential, from -37 to $-31 \mathrm{mV}$ (Fig. 4a). As the concentration increased 
further to $3 \%$, the nanoparticle size and polydispersity index decreased significantly to $103 \mathrm{~nm}$ and 0.09, respectively (Fig. 4a). Scanning electron microscopy reinforced the DLS measurements and indicated that the extent of self-assembly increased with concentration from 0.5 to $2 \%$, resulting in a shift from worm-like particles to globules and nanofibers (Fig. 4b).

\subsection{Secondary Structure Measurement}

The correlation of the silk nanoparticle secondary structure content with formulation conditions and the shear-induced assembly of silk precursor extruded through the feed needle were evaluated by attenuated total reflectance-FTIR (ATR-FTIR) analysis and deconvolution of the amide I region (Fig. S2). The structural changes caused by the nanoprecipitation process were also evaluated by

analysing the amide I region $\left(1600-1700 \mathrm{~cm}^{-1}\right)$ using the spectral correlation coefficient method. ${ }^{57}$

\subsubsection{The effect of scale and manufacturing process}

In the open semi-batch system, the silk nanoparticle $\beta$-sheet content ranged from $54-56 \%$ and compared well with the $53-56 \% \beta$-sheet content of the nanoparticles manufactured in this work using the microfluidic format. The nanoparticle secondary structure did not depend on the scale of semi-batch nanoprecipitation (Fig. 5a), and no general variation was noted in $\beta$-sheet content or correlation coefficient with extruded silk volume (Fig. S4a). By contrast, the silk nanoparticle correlation coefficient was significantly lower for nanoparticles produced at the $138 \mathrm{~mL}$ scale (0.14) than at the 1.8, 6, 21.9 and $42 \mathrm{~mL}$ scales $(0.25-0.30)$ (Fig. 5a). Additionally, the lower scale results compared more favorably with the correlation coefficients of $0.28-0.47$ obtained using the microfluidic format. 


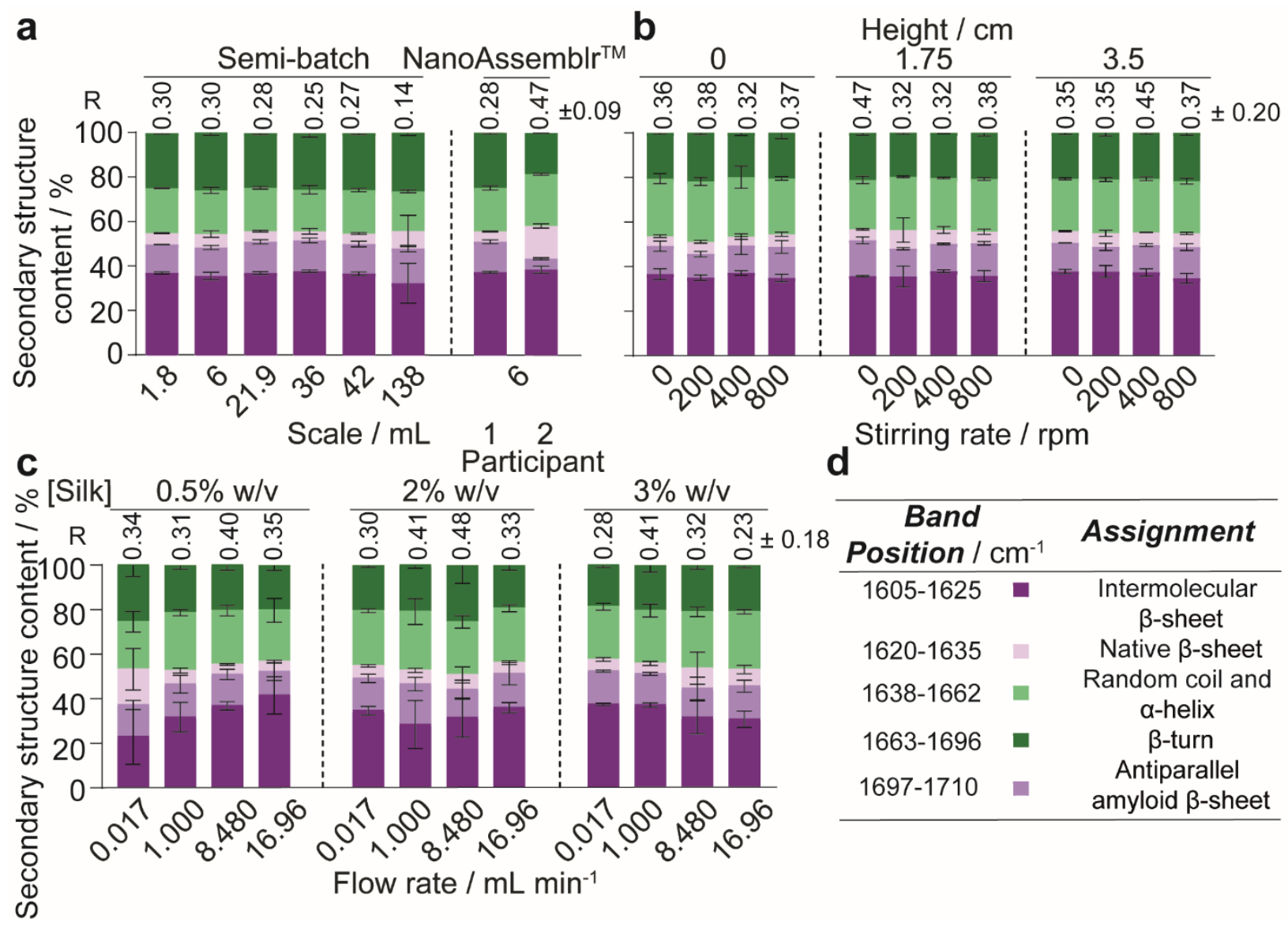

Figure 5. Formulation parameters had a significant impact on the secondary structure of silk

nanoparticles. Secondary structure content (\%) of silk nanoparticles manufactured using different (a) scales and formats of manufacture, (b) stirring rates and initial addition heights in the semibatch closed system and (c) concentrations and flow rates in the semi-batch closed system. (d) FTIR band assignments and schematic key. Secondary structure content (\%) was calculated from the relative areas of peaks in the second derivative spectrum. The correlation coefficients of silk nanoparticle, film, and powder second derivative amide I spectra were calculated using the silk II negative control film as reference. For different scales, the correlation coefficients, total $\beta$ and antiparallel amyloid $\beta$-sheet, $\beta$-turn, and $\alpha$-helix and random coil contents, were evaluated by one-way ANOVA, followed by Tukey's multiple comparison post-hoc test. The intermolecular $\beta$-sheet and native $\beta$-sheet, contents were evaluated using the Brown-Forsythe and Welch ANOVA tests, 
followed by the Dunnett T3 multiple comparison post-hoc test. Two-way ANOVA was used to compare multiple groups across feed height and stirring rate, followed by Tukey's simple effect multiple comparison post-hoc test for the correlation coefficients and contents. Two-way ANOVA was used to compare multiple groups across concentration and flow rate, followed by Tukey's pairwise multiple comparison post-hoc test for intermolecular and native $\beta$-sheet and Tukey's simple effect multiple comparison post-hoc test for anti-parallel amyloid and total $\beta$-sheet, $\alpha$-helix and random coil content, $\beta$-turn and correlation coefficients. Data obtained from semi-batch manufacture at $6 \mathrm{~mL}$ scale and silk II controls have been published elsewhere. ${ }^{41} \pm \mathrm{SD}, n=3$.

\subsubsection{The effect of flow rate, addition height and stirring rate in closed, semi-batch format}

In the unstirred closed system, increasing the feed rate from 0.017 to $7.000 \mathrm{~mL} \mathrm{~min}^{-1}$ at a $3.5 \mathrm{~cm}$ feed height caused a decrease in the $\beta$-sheet content of silk nanoparticles from $57 \%$ to $52 \%$ (Fig. S4b). Conversely, for the extruded silk, increasing the flow rate from 0.017 to $3.510 \mathrm{~mL} \mathrm{~min}^{-1}$ and $7.000 \mathrm{~mL} \mathrm{~min}^{-1}$ decreased the correlation coefficient from 0.78 to 0.44 and 0.34 , respectively (Fig. $\mathrm{S} 4 \mathrm{c})$. Further, increasing the feed height from 0.7 to $2.1 \mathrm{~cm}$ at a feed rate of $7 \mathrm{~mL} \mathrm{~min}{ }^{-1}$ decreased the nanoparticle correlation coefficient from 0.47 to 0.32 (Fig. S4b). The needle diameter had no significant effect on the nanoparticle secondary structure content (Fig. S4d); however, reducing the diameter from 1.60 to $0.33 \mathrm{~mm}$ at $7 \mathrm{~mL} \mathrm{~min}^{-1}$ increased the $\beta$-sheet content of drop-casted films from $25 \%$ to $33 \%$ (Fig. S4c). This increase in $\beta$-sheet crystallinity of the extruded silk with decreasing needle diameter was reinforced by the reduction in the correlation coefficient at feed rates of 3.510 and $7.000 \mathrm{~mL} \mathrm{~min}^{-1}$ (Fig. S4c).

In contrast to the unstirred system, the nanoparticle $\beta$-sheet content generally increased with feed height from 0.0 to $1.75 \mathrm{~cm}$ in the stirred system (Fig. 5b). For example, at $200 \mathrm{rpm}$, increasing the 
feed height from 0.0 to $1.75 \mathrm{~cm}$ increased the $\beta$-sheet content from $51 \%$ to $56 \%$ (Fig. $5 \mathrm{~b}$ ). Increasing the feed height also caused a main effect decrease in the nanoparticle $\beta$-turn content. As the stirring rate increased from 0 to $200 \mathrm{rpm}$ and then to $800 \mathrm{rpm}$, the nanoparticle antiparallel $\beta$-sheet content showed a main effect decrease and then an increase, respectively (Fig. 5b).

\subsubsection{The effect of flow rate and silk concentration in closed, semi-batch format}

The nanoparticle $\beta$-sheet content and correlation coefficients ranged between $51-58 \%$ and $0.23-$ 0.48 , respectively, and did not vary significantly with silk concentration or flow rate (Fig. 5c). However, at the low shear flow rate of $0.017 \mathrm{~mL} \mathrm{~min}^{-1}$, increasing the concentration from $0.5 \%$ to $3 \%$ reduced the nanoparticle $\alpha$-helix and random coil content from $25 \%$ to $21 \%$ and decreased the native $\beta$-sheet content from $16 \%$ to $5 \%$ (Fig. $5 \mathrm{c}$ ). The silk nanoparticle crystallinity was not affected by concentration; however, the extruded silk crystallinity increased with this factor. For instance, increasing the concentration from 2 to $3 \%$ at $8.485 \mathrm{~mL} \mathrm{~min}^{-1}$ increased the extruded silk $\beta$-sheet content from $24 \%$ to $36 \%$ (Fig. S4e). This result was paralleled by a reduction in the correlation coefficient as the concentration increased from $0.5 \%$, at flow rates of $0.017(0.90)$, $1.000(0.85)$ and $16.96 \mathrm{~mL} \mathrm{~min}^{-1}(0.69)$, to $3.0 \%$, at flow rates of $8.485(0.32)$ and $16.96 \mathrm{~mL} \mathrm{~min}^{-}$ ${ }^{1}$ (0.23) (Fig. S4e).

Increasing the flow rate from 0.017 to $16.96 \mathrm{~mL} \mathrm{~min}^{-1}$ at a $0.5 \%$ silk concentration decreased the nanoparticle native $\beta$-sheet content from $16 \%$ to $5 \%$ (Fig. $5 \mathrm{c}$ ). Likewise, the interaction between concentration and flow rate significantly reduced the nanoparticle native $\beta$-sheet content when flow rate was increased from $0.017 \mathrm{~mL} \mathrm{~min}^{-1}$ to $1 \mathrm{~mL} \mathrm{~min}^{-1}$ and $16.96 \mathrm{~mL} \mathrm{~min}^{-1}$ at silk concentrations of $0.5 \%, 2 \%$ and $3 \%$, respectively (Fig. $5 \mathrm{c}$ ). In contrast to the nanoparticle structural changes, the $\beta$-sheet content of $0.5 \%$ silk increased with flow rate from $19 \%$ at $0.017 \mathrm{~mL} \mathrm{~min}^{-1}$ to $31 \%$ at $8.485 \mathrm{~mL} \mathrm{~min}{ }^{-1}$ (Fig. S4e). The interaction between flow rate and concentration also 
significantly increased the silk $\beta$-sheet content, from $19 \%$ to $29 \%$ and $37 \%$, as the flow rate was increased from $0.017 \mathrm{~mL} \mathrm{~min}{ }^{-1}$ to $16.96 \mathrm{~mL} \mathrm{~min}^{-1}$ and $16.96 \mathrm{~mL} \mathrm{~min}{ }^{-1}$ at silk concentrations of $0.5 \%, 2.0 \%$ and $3.0 \%$, respectively (Fig. S4e). These results were supported by the reduction in the correlation coefficient of extruded silk from 0.78 to 0.32 and 0.23 as the flow rate increased from $0.017 \mathrm{~mL} \mathrm{~min}^{-1}$ to 8.485 and $16.96 \mathrm{~mL} \mathrm{~min}^{-1}$ at a silk concentration of $3.0 \%$ (Fig. S4e).

\subsection{Thermal analysis}

\subsubsection{The effect of scale}

Table 1 and Fig. 6 show the first-cycle simultaneous thermal analysis results for the silk nanoparticles manufactured in the open, semi-batch system at scales between 1.8 and $138 \mathrm{~mL}$ versus the silk II negative controls. TGA of the residual water contents and the thermal stabilities of the silk nanoparticles showed a loss of adsorbed and strongly bound water between 20 and 140 ${ }^{\circ} \mathrm{C}$ and silk decomposition above $170{ }^{\circ} \mathrm{C}$. Nanoparticles manufactured at scales between 6 and 138 $\mathrm{mL}$ had a water content of $10.7-13.0 \%(\mathrm{w} / \mathrm{w})$, and this increased significantly with scale. The onset decomposition temperature of the silk nanoparticles manufactured at these scales ranged between $271.5^{\circ} \mathrm{C}$ and $278.1^{\circ} \mathrm{C}$ and was significantly higher than that of the negative silk II control $\left(261.4{ }^{\circ} \mathrm{C}\right)$. This result confirmed the higher content of crystalline $\beta$-sheet structures in the silk nanoparticles compared to the silk I polymorph present in freeze-dried silk. An increase in the manufacturing scale from 6 and 21.9 to $138 \mathrm{~mL}$ also caused a significant decrease in the onset decomposition temperatures of the silk nanoparticles. 
Table 1. First cycle thermal properties of silk nanoparticles manufactured at multiple scales in open semi-batch format.

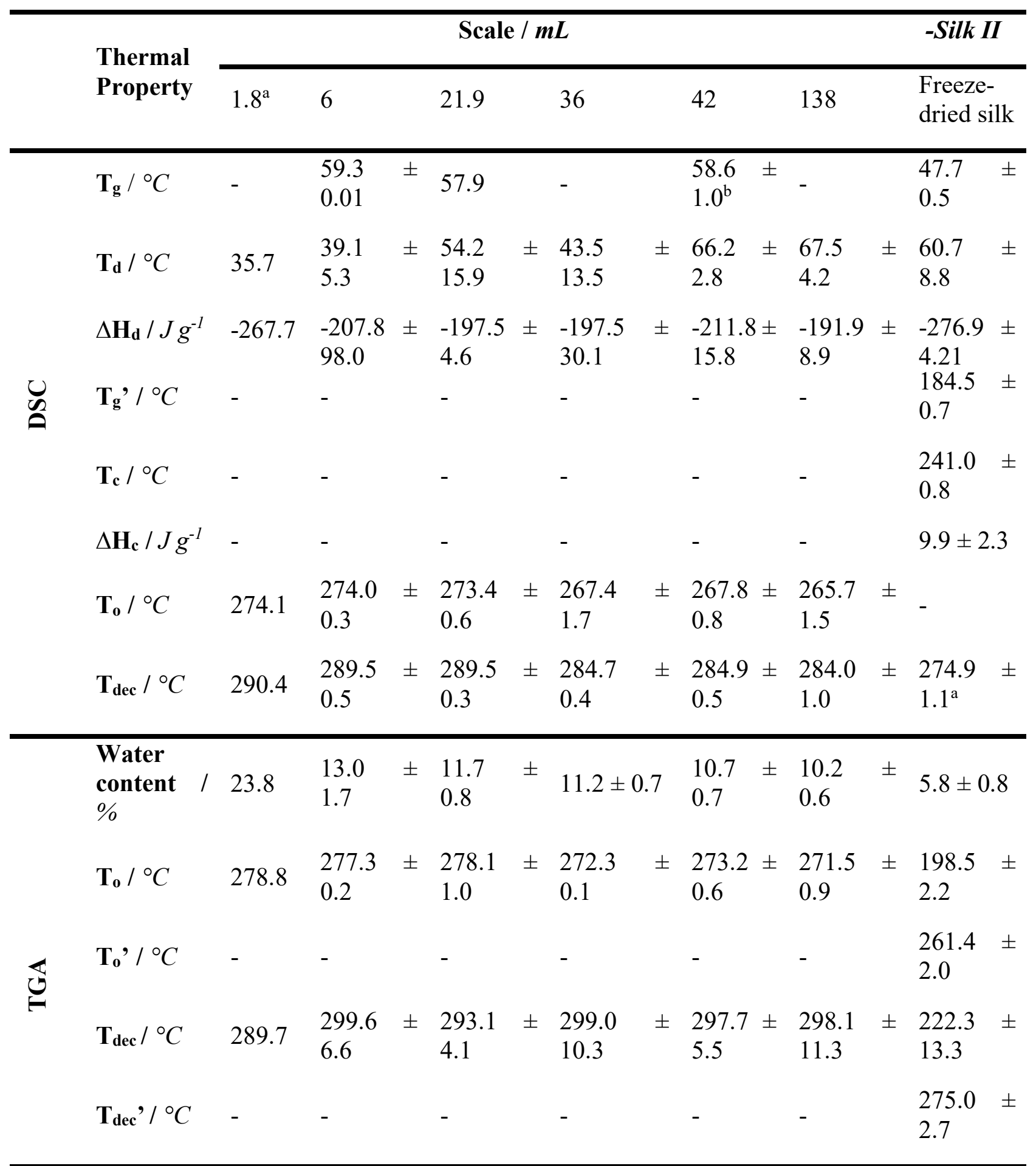

a. $n=1$ 
a

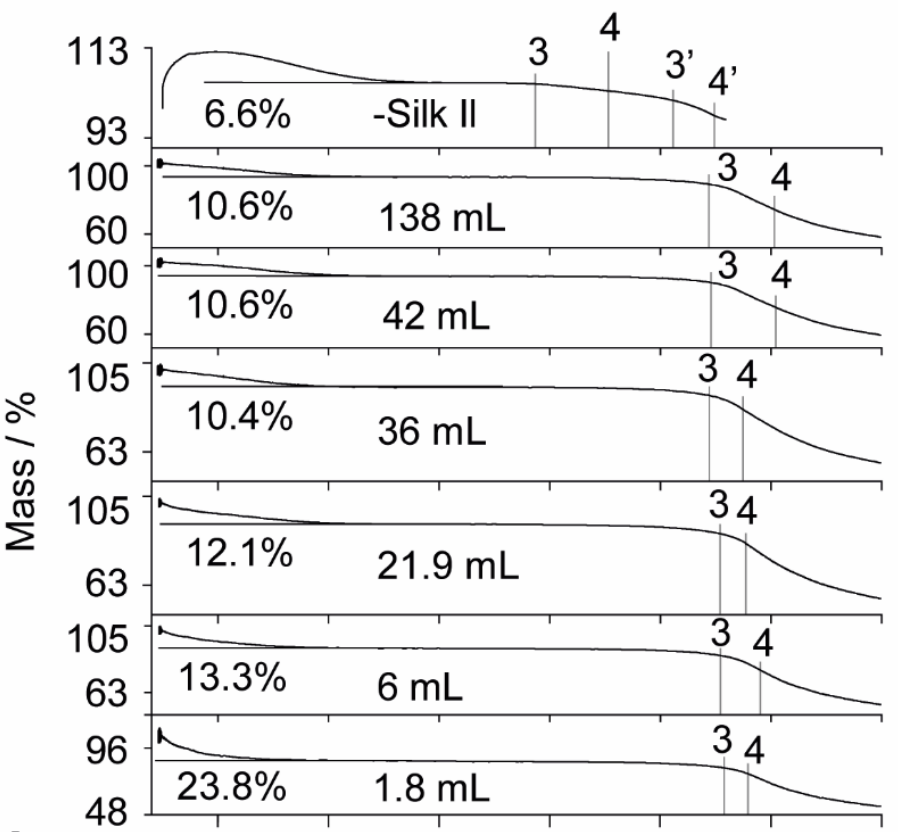

b

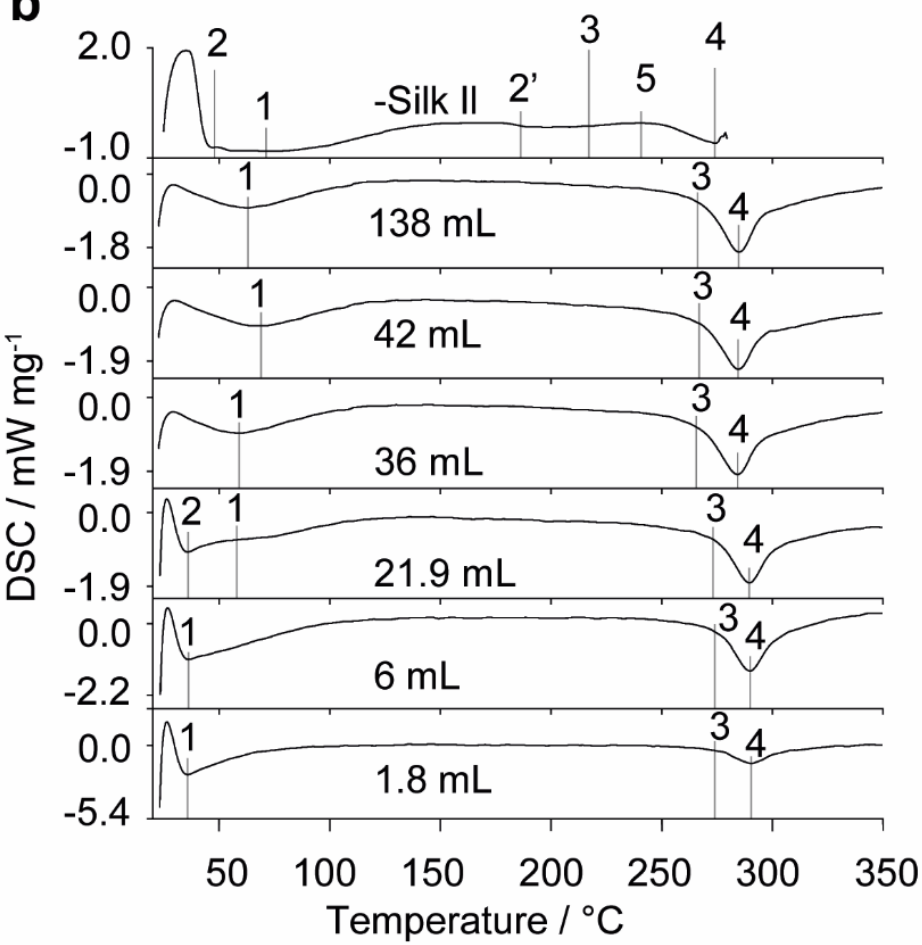

C

\begin{tabular}{cc}
\hline Notation & Thermal event \\
\hline 1 & $\mathrm{~T}_{\mathrm{d}}$ \\
2 & $\mathrm{~T}^{\mathrm{g}}$ \\
3 & $\mathrm{~T}^{\mathrm{o}}$ \\
4 & $\mathrm{~T}_{\mathrm{dec}}$ \\
5 & $\mathrm{~T}_{\mathrm{c}}$ \\
\hline
\end{tabular}


extrapolated onset temperature of crystallization and decomposition $\left(\mathrm{T}_{\mathrm{o}}\right)$, crystallization temperature $\left(\mathrm{T}_{\mathrm{c}}\right)$ and decomposition temperatures $\left(\mathrm{T}_{\mathrm{dec}}\right)$ are reported. Data obtained from silk II controls have been published elsewhere. ${ }^{41}$

The secondary structure and thermal stability of the silk nanoparticles were also evaluated by differential scanning calorimetry (Fig. 6). The desorption enthalpy required to remove adsorbed water did not vary with scale, although the desorption enthalpy was significantly higher for the nanoparticles manufactured at the $138 \mathrm{~mL}$ scale $\left(-191.9 \mathrm{~J} \mathrm{~g}^{-1}\right)$ than for the negative control $(-276.9$ $\mathrm{J} \mathrm{g}^{-1}$ ). The temperature of desorption ranged from 36.0 to $67.5^{\circ} \mathrm{C}$, and it increased significantly from $39.1{ }^{\circ} \mathrm{C}$ to $66.2{ }^{\circ} \mathrm{C}$ and $67.5^{\circ} \mathrm{C}$ when the scale was increased from $6 \mathrm{~mL}$ to $42 \mathrm{~mL}$ and 138 $\mathrm{mL}$. Increasing the manufacturing scale from 6 to $42 \mathrm{~mL}$ also caused a significant decrease in the onset decomposition temperatures of silk nanoparticles from 274.0 to $267.8{ }^{\circ} \mathrm{C}$, and this temperature declined further as the scale increased from 21.9 to $138 \mathrm{~mL}$ (from $273.4{ }^{\circ} \mathrm{C}$ to 265.7 $\left.{ }^{\circ} \mathrm{C}\right)$. The decomposition temperatures followed the same trend with increasing scale, decreasing from $289.5^{\circ} \mathrm{C}$ at $6 \mathrm{~mL}$ to $284.7^{\circ} \mathrm{C}$ at $36 \mathrm{~mL}$, but then levelled off as scale was increased further, up to $138 \mathrm{~mL}\left(284.0^{\circ} \mathrm{C}\right)$.

\subsection{Silk nanoparticle aqueous stability}

\subsubsection{The effect of scale and manufacturing process}

The aqueous stability of the nanoparticles manufactured in the open, semi-batch system and in the staggered herringbone micromixer at scales between 1.8 and $138 \mathrm{~mL}$ were determined for up to 42 days by DLS and ELS (Fig. 7). Nanoparticles manufactured across all scales and formats showed size stability in water for the entire duration of the study (Fig. 7a), and the polydispersity index and zeta potential measurements remained consistent for nanoparticles produced in semi- 
batch format at scales above $21.9 \mathrm{~mL}$ and in microfluidic format (Fig. $7 \mathrm{~b}$ and c). Conversely, the size polydispersity of the nanoparticles manufactured at the $1.8 \mathrm{~mL}$ scale in semi-batch format increased significantly at 10 days, but did not significantly differ from the initial measurement thereafter. The zeta potential of the nanoparticles manufactured at the 1.8, 6 and $21.9 \mathrm{~mL}$ scales also fluctuated across the 42 days.

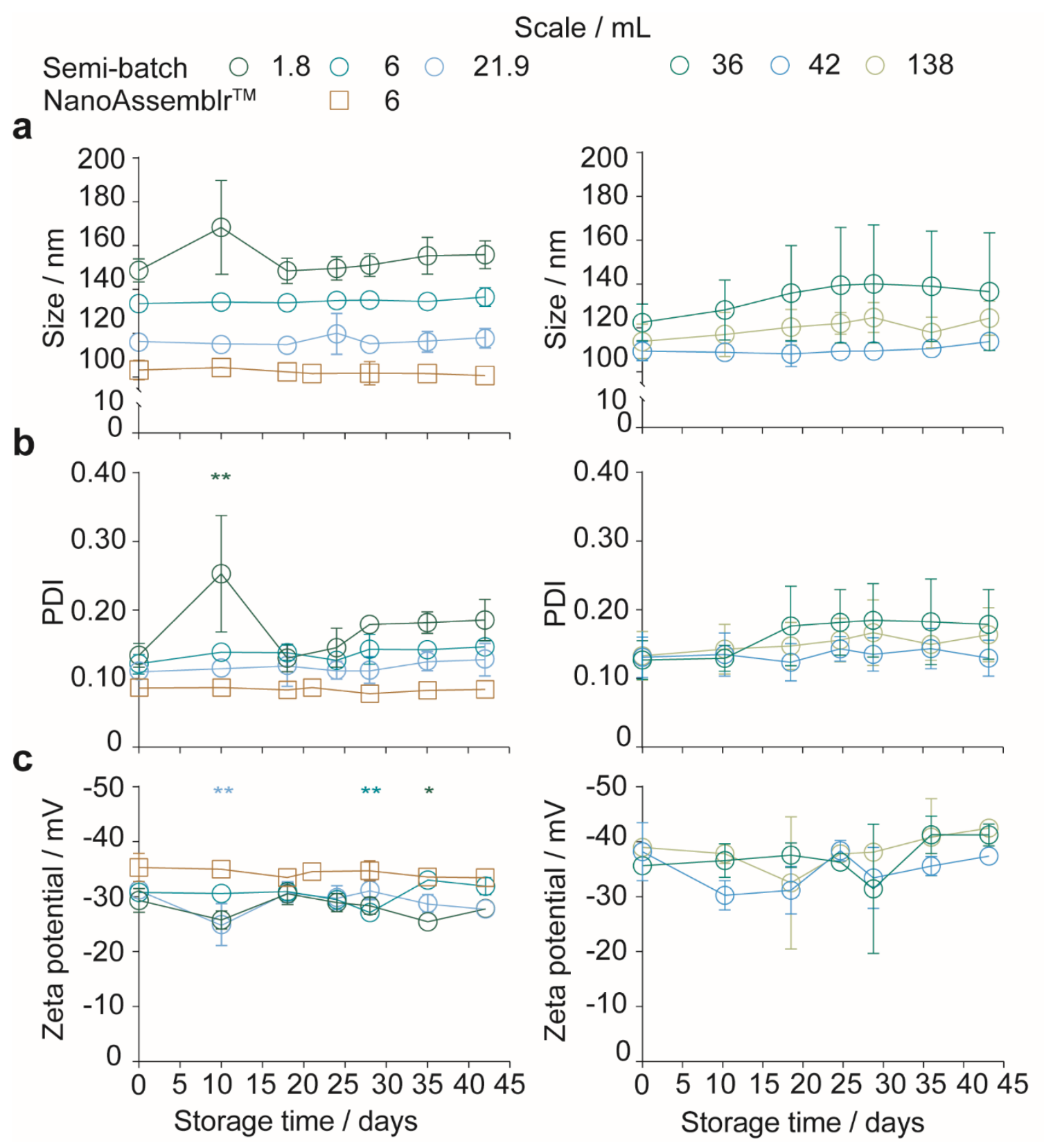


Figure 7. Stability of silk nanoparticles manufactured in drop-by-drop semi-batch and microfluidic formats by varying scale. (a) Hydrodynamic diameter, (b) polydispersity index (PDI), (c) zeta potential of silk nanoparticles stored in water at $4{ }^{\circ} \mathrm{C}$. Diluted nanoparticle suspensions were vortexed prior to DLS analysis, $\pm \mathrm{SD}, n=3$. Unless otherwise stated, the silk nanoparticle stability was evaluated by one-way ANOVA followed by Dunnett's post hoc test to compare between $t=0$ day control and $t>0$ day samples. The zeta potential stability of nanoparticles manufactured at 36 and $138 \mathrm{~mL}$ scale were evaluated using the Brown-Forsythe and Welch ANOVA followed by Dunnett's T3 post hoc test. Asterisks denote statistical significance for each formulation between $\mathrm{t}=0$ and $\mathrm{t}>0$ days, determined using post-hoc tests as follows: ${ }^{*} \mathrm{p}$ $<0.05, * * \mathrm{p}<0.01$

\section{Discussion}

In the current study, the optimised formulation variables ${ }^{11-13}$ for preparing silk nanoparticles were first used to compare the controllability of the mixing processes in semi-batch and continuous formats. We used the open, semi-batch process and active mixing for lab-scale nanoprecipitation, and the NanoAssemblr ${ }^{\mathrm{TM}}$ platform for the continuous process, with a commercially available staggered herringbone mixer operating under conditions of laminar flow $\left(\mathrm{R}_{\mathrm{e}}<2000\right)$.

\subsection{The effects of the scale and manufacturing process}

The scaling issue of semi-batch nanoprecipitation is well-documented. ${ }^{59,60}$ Operation of the semibatch open system was undertaken at a range of scales appropriate for lab-scale applications. The low flow rate ensured laminar flow in the syringes and needle; the maximum wall shear rate in the syringes was estimated to be between 8.9 and $261 \mathrm{~ms}^{-1}$, while the wall shear rate in the needle was estimated as $4724 \mathrm{~s}^{-1}$. Combined with the low residence time of $33 \mathrm{~ms}$, these shear rates would not 
be expected to provide sufficient work $\left(\approx 10^{5} \mathrm{~Pa}\right)^{14}$ for shear-induced nucleation of the silk molecules. In addition, the $3 \% \mathrm{w} / \mathrm{v}$ concentration used at all scales fell below the $\approx 10 \% \mathrm{w} / \mathrm{w}$ critical micelle concentration ${ }^{42}$ of regenerated silk fibroin. This was confirmed by the lack of correlation between the volume of extruded silk and the $\beta$-sheet content or correlation coefficient. Consequently, the following discussion assumes homogeneous nucleation induced by desolvation and does not consider shear-induced nucleation followed by seeded crystallization (Fig. 8). 


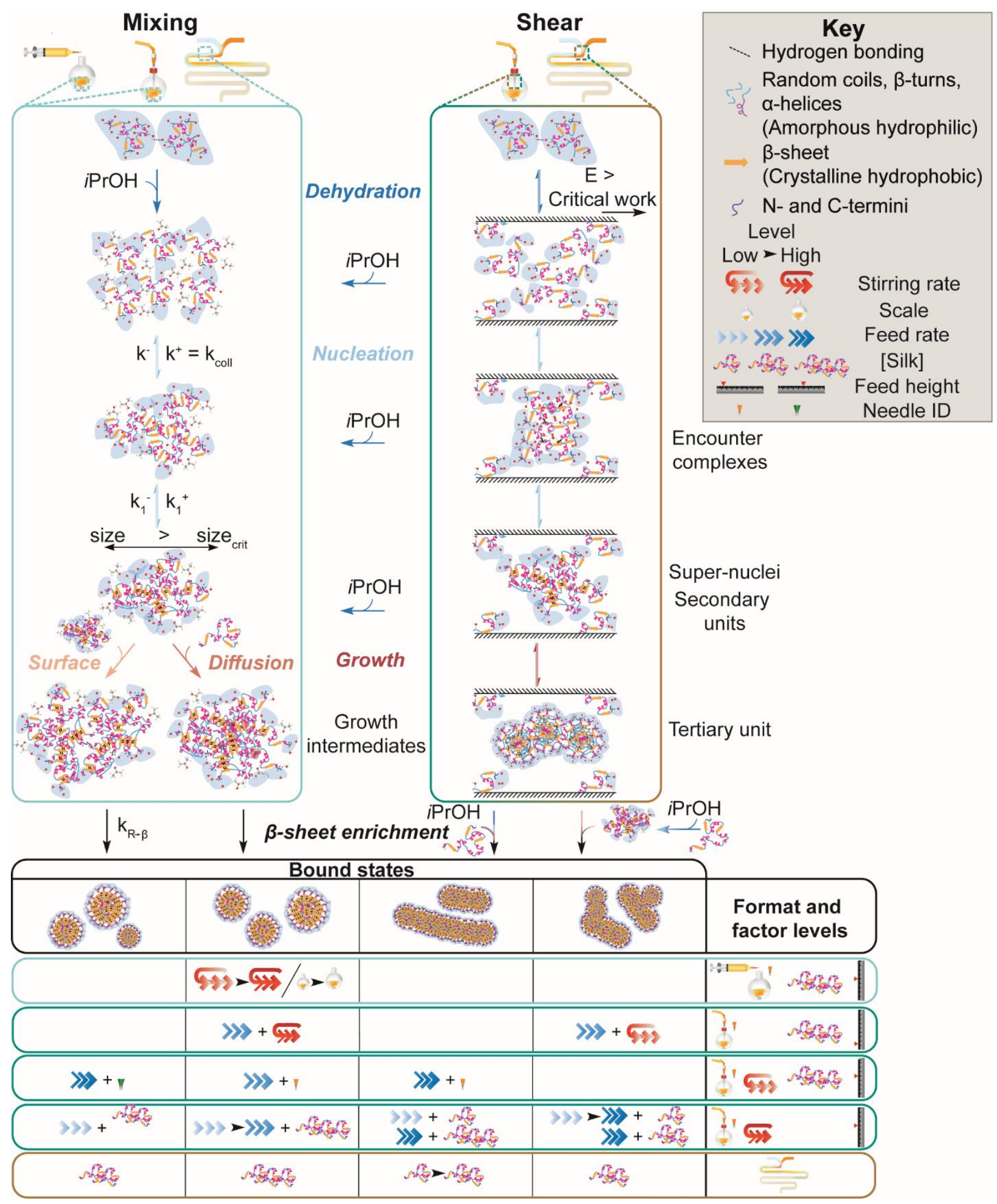

Figure 8. Schematic of protein-protein association and $\beta$-sheet assembly of silk fibroin via anti-solvent and shear-induced desolvation. The mechanism under shear flow is adapted from 
Dunderdale et al. ${ }^{14}$ and Zhang et al. ${ }^{61}$ Silk molecules, nanoparticles and stoichiometry of association are not drawn to scale.

Keeping all other factors constant but increasing the total volume resulted in a decrease in the nanoparticle size and zeta potential, although the polydispersity index and yield did not change significantly (Fig. 2a). Numerous studies with polymer and protein nanoprecipitation have indicated that particle size decreases with increasing mass fractions of anti-solvent. ${ }^{62}$ In the present study, maintaining a constant droplet volume while increasing the volume of the anti-solvent caused a decrease in the initial volume ratio of silk to anti-solvent decreasing from $25 \times 10^{-3}$ at 1.8 $\mathrm{mL}$ to $0.32 \times 10^{-3}$ at $138 \mathrm{~mL}$. Studies with polymeric and protein nanoprecipitation have indicated that the particle size decreases with increasing mass fractions of anti-solvent. ${ }^{62}$ In solvent mixtures containing a higher mass fraction of anti-solvent, the solute equilibrium concentration is reduced, thereby increasing supersaturation along with the rate and degree of nucleation. The reduction in surface-controlled nuclei and in particle growth rates with local solute concentration causes a shift to a diffusion-limited growth regime. Growth is disfavored as the distance required for diffusion increases with the anti-solvent volume, thereby reducing the likelihood of silk association. Finally, the Reynolds number and turbulence during droplet addition increased with anti-solvent volume and this decreased the micromixing time.

We propose that stirring at small and large scales is a simple technique for maintaining reproducibility when increasing the total volume and keeping all other factors constant. This is important for formulation screening which is typically conducted on a small scale. The physicochemical properties were made more similar with the 6 -fold scale-up from 6 to $36 \mathrm{~mL}$ by increasing the stirring rate to $400 \mathrm{rpm}$ and thereby reducing the mixing times from $>120 \mathrm{~s}$ to 10.1 
and $41.8 \mathrm{~s}$, respectively (Fig. 1c). Compared to the significant reduction in nanoparticle size with stirring at the $6 \mathrm{~mL}$ scale, the nanoparticle size decreased only slightly with stirring at the $36 \mathrm{~mL}$ scale. First, at a larger scale and in the absence of stirring, an increased degree of solvent and antisolvent mixing could occur during silk addition until the critical nucleation concentration was reached, thereby enabling faster nucleation rates and the formation of smaller particles. Second, the standard deviation of the particle size was relatively high for both stirred and unstirred processes. This reflects the slightly different feed point positions, which had a greater effect on the fluid dynamics and mixing times at larger volumes. The lack of change in the polydispersity index with mixing time or scale suggests that growth was operating under diffusion-limited or mononuclear surface control.

The secondary structure content was not significantly affected by the change in supersaturation throughout scale-up, although a slight reduction in the correlation coefficient occurred for nanoparticles manufactured at $138 \mathrm{~mL}$ scale. This reflected a slightly reduced intermolecular $\beta$ sheet content and a higher native $\beta$-sheet content compared to those of nanoparticles produced at smaller scales. The scale-up caused no significant difference in water content or in desorption enthalpy, but the temperature of the desorption increased and the desorption endotherm broadened with increasing scale (Table 1, Fig. 6). This indicated that the types of water binding modes within the nanoparticle structure may have increased with the scale of manufacture. Simultaneous thermal analysis showed a significant reduction in the thermal stabilities of nanoparticles manufactured at increasing scale (Table 1, Fig. 6). This finding indicated that either the degree of intermolecular $\beta$-sheet formation was reduced ${ }^{63}$ or the silk molecules that were incorporated into nanoparticles manufactured at a higher scale had a lower weight and length distribution. ${ }^{13}$ 
Continuous manufacture is well recognized as simplifying the scale-up process of the nanoformulations ${ }^{64}$ so manufacture using the optimized formulation parameters in the NanoAssemblr ${ }^{\mathrm{TM}}$ was used as a scale-independent control. Assuming Newtonian flow, the $1 \mathrm{~mL}$ $\min ^{-1}$ flow rate resulted in a Reynolds number of 40 , which was below the high vorticity and low transverse flow regime (Reynolds numbers $>1000$ ), thereby ensuring high mixing efficiency. The residence time was longer than the mixing time (Fig. 1c), indicating that complete mixing occurred in the micromixer.

The wall shear rate $\left(80114 \mathrm{~s}^{-1}\right)$ was used as an estimate for the maximum shear rate experienced in the micromixer. When combined with the residence time, the shear-induced nucleation of silk fibroin was probable, as both the critical shear rate ${ }^{65}$ and work ${ }^{14}$ were exceeded. Shear-induced nucleation was supported by a previous demonstration that an increase in the flow rate from 1 to $12 \mathrm{~mL} \mathrm{~min}^{-1}$ (shear rate $961499 \mathrm{~s}^{-1}$ ) reduced particle curvature and increased the overall size. ${ }^{12}$ Hence, contrary to homogeneous nucleation in semi-batch format, the micromixer provided a seeded crystallization process (Fig. 8).

Regenerated silk fibroin undergoes a primary assembly process into silk fibroin micellar structures (secondary building units) (Fig. 8). ${ }^{61}$ The hydrophilic blocks of the silk fibroin polymer are hydrated in aqueous solution, resulting in an extended conformation. ${ }^{61}$ The shear-induced desolvation breaks the stabilizing intermolecular bonds with water and enables hydrophobic interactions between silk molecules. ${ }^{61}$ For the optimized formulation parameters, the work supplied in the microchannel was probably not sufficient for secondary assembly ${ }^{61}$ to occur in the desolvating layer prior to complete blending of the binary mixture. Following complete mixing, kinetic locking of the spherical silk nanoparticle structure would be expected due to the transition from a random coil to a $\beta$-sheet secondary structure. 
Using the NanoAssemblr ${ }^{\mathrm{TM}}$ format, scale-up can be achieved by chip parallelization and increasing the total flow rate. In this way, the fluid dynamics and solution composition in the micromixer are independent of scale. However, special cause variation can be introduced by slight differences in the microfluidic chip dimensions. Hence, the reproducibility limit from the round robin study could be used as a general guide for the differences expected between nanoparticles manufactured using multiple chips (Table S4, Figure S1). Importantly, silk nucleation in the feed lines could decrease the reproducibility, thereby limiting the maximum total flow rate and reducing the scalability of continuous manufacture. For this reason, the feed line dimensions should be selected to reduce the wall shear rate while maintaining laminar flow.

\subsection{The effect of flow rate, addition height and stirring rate in closed, semi-batch format}

The flow rates of $0.017,3.51$ and $7 \mathrm{~mL} \mathrm{m^{-1 }}$ resulted in Reynolds numbers $<2000$ within the tubing and with all needles used; hence, laminar and Newtonian flow was used to calculate the average wall shear rate. ${ }^{42}$ The average wall shear rates in the syringe and tubing (Fig. 1), combined with the residence times, were not expected to induce silk nucleation at flow rates between 0.017 and $7.000 \mathrm{~mL} \mathrm{~min}^{-1}$. However, conditions of high shear were introduced at flow rates above 0.017 $\mathrm{mL} \mathrm{min}^{-1}$ in needles with diameters less than $1.6 \mathrm{~mm}$ (Fig. 1). Scanning electron microscopy showed that shear-induced nucleation resulted in secondary and tertiary assembly of silk micelles into globules and nanofibers, prior to anti-solvent addition and completion of mixing (Fig. S3c, Fig. $3 \mathrm{~b}$ and Fig. 4b). ${ }^{66}$ This was reinforced by the higher $\beta$-sheet content of the silk extruded from the $0.33 \mathrm{~mm}$ needle compared to the $1.60 \mathrm{~mm}$ needle at $7 \mathrm{~mL} \mathrm{~min}^{-1}$ (Fig. S4c). Additionally, the silk extruded from the $0.33 \mathrm{~mm}$ needle showed significantly lower correlation coefficients at all flow rates above $0.017 \mathrm{~mL} \mathrm{~min}^{-1}$ (Fig. S4c). 
Without stirring, at a $1 \mathrm{~mL} \mathrm{~min}{ }^{-1}$ feed rate and $0.0 \mathrm{~cm}$ height, the shear-induced nucleation and the anti-solvent-induced dehydration resulted in nanoparticle formation at the phase boundary upon addition of the aqueous silk feed to the isopropanol bulk. This resulted in phase separation and low reproducibility due to the uncontrolled manual mixing of the two phases during purification. Increasing the stirring rate and feed height increased the rate and degree of antisolvent-induced nucleation by reducing the mixing time (Fig. 1c). Assembly growth was disfavored under conditions of low mixing time, resulting in low nanoparticle size and polydispersity with increased yield. Additionally, the shear stress of the liquid flow arising from stirring rates and feed heights of $200 \mathrm{rpm}$ and $1.75 \mathrm{~cm}$ could exceed the shear stress of agglomerates formed during silk extrusion from the feed needle. Breaking the intermolecular bridges holding the agglomerates together, combined with desolvation, would result in kinetic locking of the secondary building units. This was supported by the increase of nanoparticle $\beta$-sheet content with feed height (Fig. 5b) and the low curvature morphology of tertiary assemblies formed at low heights and stirring rates (Fig. 3b).

\subsection{The effect of flow rate and concentration in closed, semi-batch and microfluidic formats}

In the micromixer, the low anti-solvent-induced nucleation rate at the $0.5 \%$ silk concentration resulted in diffusion-limited or polynuclear growth of worm-like assemblies (Fig. 4b). Association

and assembly processes were favored by increasing silk concentration from 0.5 to $2 \%$, ${ }^{61}$ resulting in larger worm-like tertiary structures with a higher polydispersity index (Fig. 4). The increased degree of shear-induced nucleation at $2 \%$ concentration reduced supersaturation and increased the nucleation energy barrier. As the viscosity increased with primary and secondary assembly, ${ }^{65}$ the energy barrier increased further. This resulted in a lower rate and degree of anti-solvent nucleation and a longer growth regime of micelles in the desolvating layers. 
As the silk concentration increased from 2 to $3 \%$, the viscosity increased gradually and the surface tension decreased rapidly, ${ }^{65,67}$ which resulted in a reduction of the water transport rate between the aqueous and isopropanol phases. This may have caused the mixing time to decrease, providing less time for shear-induced secondary self-assembly by kinetically freezing the micellar structure. Finally, the reduction in the critical shear rate between 2 and $3 \%{ }^{65}$ increased the rate and degree of shear-induced nucleation. This disfavored anti-solvent induced nucleation and growth and resulted in small, spherical nanoparticles of a narrow size distribution, produced in high yield. $^{61}$

In the stirred semi-batch process, at feed rates of $0.017,1.000,8.485$ and $16.96 \mathrm{~mL} \mathrm{~min}^{-1}$, the shear rates experienced by silk in the fluid line were estimated as $0.18,10,88$ and $176 \mathrm{~s}^{-1}$, respectively, while the shear rates in the needle were estimated as $80,4724,40083$ and $80114 \mathrm{~s}^{-1}$ (Table S2, Fig. 1b). At $1 \mathrm{~mL} \mathrm{~min}^{-1}$ and above, the needle shear rates lay above the critical shear rate and work was required for nucleation. ${ }^{65}$ Consequently, the following arguments assume that shear-induced nucleation, followed by isopropanol-induced desolvation, occurred above a flow rate of $0.017 \mathrm{~mL} \mathrm{~min}^{-1}$. Shear-induced nucleation was reinforced by a decrease in the correlation coefficients of extruded silk as the flow rate increased across all concentrations and an increase in the $\beta$-sheet content of extruded silk as the flow rate increased at 0.5 and $2.0 \%$ (Fig. S4e).

For homogenous nucleation at $0.017 \mathrm{~mL} \mathrm{~min}^{-1}$, increasing the concentration from 0.5 to $3.0 \%$ reduced the assembly size and polydispersity index due to a greater degree of supersaturation (Fig. 4). Samples could be considered monodisperse using 3.0\% silk at flow rates of 0.017 and 1.000 $\mathrm{mL} \mathrm{min}^{-1}$. At $0.017 \mathrm{~mL} \mathrm{~min}^{-1}$, the reduction in the growth phase with increasing concentration significantly increased assembly curvature (Fig. 4b) and packing, as native $\beta$-sheet, $\alpha$-helix and random coil contents decreased (Fig. 5c). 
Under high shear conditions at all flow rates exceeding $0.17 \mathrm{~mL} \mathrm{~min}{ }^{-1}$, increasing the concentration from 0.5 to $3.0 \%$ did not significantly reduce the particle size (Fig. 4a) or the extent of silk assembly (Fig. 4b). This was possibly due to a balance between reduced silk-anti-solvent mixing times and increased rates of shear-induced self-assembly with concentration. Consequently, as the number of shear-induced micelles formed increased with feed rate, the reduced silk concentration prohibited anti-solvent-induced nucleation. The greater degree of shear-induced self-assembly with increasing concentration, from 0.5 to $3.0 \%$, was reinforced by the increase in $\beta$-sheet content of extruded silk and the reduction of correlation coefficients at 8.485 and $16.96 \mathrm{~mL} \mathrm{~min}^{-1}$ (Fig. S4e).

The feed rate of silk also controls the degree and rate of supersaturation onset of the anti-solventinduced desolvation. This means that the type and rate of anti-solvent-induced nucleus growth could be impacted by the rate of silk addition. Increasing the feed rate favors mononuclear surfacecontrolled growth by increasing the mass transfer of silk from the solution to the assembly surface. As the feed rate increased, this resulted in assemblies being produced with greater size at $2.0 \%$ and size distributions increasing at 0.5 and $2.0 \%$ (Fig. 4a). This agrees with the outcome of BSA

nanoprecipitation, ${ }^{59}$ but contrasts with the results obtained with other nanoparticle precursors, such as curcumin, where increasing the solute addition rate reduced the resulting nanoparticle sizes due to greater mixing efficiency. ${ }^{62}$

\subsection{Stability of nanoparticles manufactured in semi-batch and microfluidic formats}

A large nanoparticle surface area is beneficial for biomedical applications, but it is responsible for a high surface energy, which can result in a metastable nanoparticle structure. In the absence of sufficient steric and electrostatic repulsion between nanoparticles, the surface energy is lowered by particle agglomeration and flocculation. Nanoparticles proposed for intravenous administration 
need to exhibit stability to standard storage conditions to prevent clinical problems (e.g., inaccurate dosing and impeded blood flow). The characterization of the effect of ageing on nanoparticle physicochemical properties is important for maximizing shelf life and preventing undesired complications. For this reason, we also examined the long-term stability of silk nanoparticles manufactured in the open, semi-batch system and the NanoAssemblr ${ }^{\mathrm{TM}}$ for 45 days at $4{ }^{\circ} \mathrm{C}$ to assess storage capabilities (Fig. 7).

Similar to previous studies, ${ }^{11-13}$ the zeta potential of nanoparticles from all formulations on the day of manufacture was lower than $-25 \mathrm{mV}$, at $\mathrm{pH} \sim 7.4$. This indicated the presence of sufficient electrostatic repulsion between particles for moderate aqueous stability. Indeed, all silk nanoparticles manufactured showed size stability over the entire study period. Fluctuations in polydispersity and zeta potential occurred for nanoparticles produced from some semi-batch formulations, and while these changes were significant, they did not follow a trend indicative of time-dependent flocculation or coagulation. ${ }^{12}$ Dissolution also did not occur, as the size and polydispersity remained relatively constant throughout the study.

\section{Conclusion}

The flow properties of silk fibroin are fundamental to the function of the biopolymer in the natural world and can be exploited in nanoprecipitation. Here, to aid the progress of silk nanomedicines from bench to market and to improve in vitro and in vivo therapeutic profiling of silk nanomedicines, we studied the impact of semi-automated manufacture of silk fibroin nanoparticles on their properties and process reproducibility by comparing high shear microfluidic processes with high and low shear semi-batch processes. Scalable semi-batch nanoprecipitation enables inter-laboratory and manufacturing format comparisons. We found that under homogeneous nucleation, as the scale was increased, the particle size and surface charge decreased $(138 \mathrm{~mL}<$ 
$1.8 \mathrm{~mL}$ ), due to an increase in supersaturation and reduced mixing times. Greater reproducibility on scale-up could therefore be obtained with active stirring at $400 \mathrm{rpm}$ in semi-batch format and using high shear, microfluidic-assisted manufacture.

In a high shear, semi-batch closed system, we showed that, at flow rates where the critical shear rate was exceeded, the nanoparticle size, polydispersity, crystallinity, and zeta potential magnitude decreased and the yield increased significantly with mixing time, as the stirring rate $(800,400<$ $200<0 \mathrm{rpm})$ and feed addition height $(3.5<1.75<0 \mathrm{~cm})$ increased. At the optimised addition height and stirring rate, the shear-induced nucleation and surface-controlled growth resulted in increases in size and polydispersity as the feed addition rate increased $(0.017<1.000<8.485<$ $\left.16.96 \mathrm{~mL} \mathrm{~min}^{-1}\right)$. Increasing concentration reduced the nanoparticle size under homogeneous nucleation $(0.5 \%>2.0 \%>3.0 \%)$ and the polydispersity decreased under both seeded and homogenous crystallization due to increased supersaturation. Conversely, in the high shear microfluidic process, where the critical shear rate was exceeded, we showed that nanoparticle size, polydispersity and zeta potential were controlled by concentration $(0.5 \%<2.0 \%>3.0 \%)$. The information provided in this study delineates rational guidelines for the preparation of silk fibroin nanoparticles under shear and anti-solvent-induced desolvation by increasing supersaturation and reducing the mixing time.

\section{ASSOCIATED CONTENT}

\section{Supporting Information}

The Supporting Information is available free of charge on the ACS Publications website. 
Flow characteristics of semi-batch system, round robin results in microfluidic format, the impact of flow rate, feed height and needle diameter formulation parameters on silk nanoparticle characteristics, exemplary smoothed second derivative FTIR spectra and peak fitting in the amide I region for nanoparticles, films and powders, secondary structure contents and correlation coefficients of silk nanoparticles and extruded silk films manufacture using the semi-batch system (PDF).

\section{AUTHOR INFORMATION}

\section{Corresponding Author}

* Corresponding author: Tel: +44 (0) 141548 2510; E-mail: philipp.seib@strath.ac.uk.

\section{Author Contributions}

S.A.L.M. designed, collected, analyzed, and interpreted the data and generated the manuscript draft. R.R. conducted silk concentration experiments using microfluidics and interpreted results. Y.P. and F.P.S. provided training, advised on experimental design, and contributed to the interpretation of the results. All authors discussed the results and/or provided advice on the experimental analysis. F.P.S. supervised the project and content-edited the manuscript.

\section{Funding Sources}

S.A.L.M. is supported by a Medical Research Scotland Ph.D. Studentship (PhD-1292-2018).

\section{Notes}

The authors declare no competing financial interest.

All data supporting this research are openly available from 


\section{ACKNOWLEDGMENTS}

The authors thank Professor Andrea Ducci (University College London, England, U.K.), Dr Alice Turner, Dr Deborah Bowering and Dr Maider Olasolo (University of Strathclyde, Scotland, U.K.) for providing training and technical advice. The authors acknowledge that this work was carried out in part at the EPSRC Future Manufacturing Research Hub for Continuous Manufacturing and Advanced Crystallisation (CMAC) (EP/P006965/1) and was supported by a U.K. Research Partnership Fund award from the Higher Education Funding Council for England (Grant HH13054). The authors acknowledge that the electron scanning microscopy work was carried out at the Advanced Materials Research Laboratory, housed within the University of Strathclyde.

\section{ABBREVIATIONS}

Aq., aqueous; DSC, dynamic scanning calorimetry; PDI, polydispersity index; SEM, scanning electron microscopy; TGA, thermogravimetric analysis.

\section{REFERENCES}

(1) Konwarh, R. Can the Venerated Silk Be the Next-Generation Nanobiomaterial for Biomedical-Device Designing, Regenerative Medicine and Drug Delivery? Prospects and Hitches. Bio-Des. Manuf. 2019, 2, 278-286. DOI: 10.1007/s42242-019-00052-9.

(2) Chouhan, D.; Mandal, B. B. Silk Biomaterials in Wound Healing and Skin Regeneration Therapeutics: From Bench to Bedside. Acta Biomater. 2020, 103, 24-51. DOI: 10.1016/j.actbio.2019.11.050. 
(3) Maitz, M. F.; Sperling, C.; Wongpinyochit, T.; Herklotz, M.; Werner, C.; Seib, F. P. Biocompatibility Assessment of Silk Nanoparticles: Hemocompatibility and Internalization by Human Blood Cells. Nanomedicine: NBM 2017, 13, 2633-2642. DOI: 10.1016/j.nano.2017.07.012.

(4) Lozano-Pérez, A. A.; Montalbán, M. G.; Aznar-Cervantes, S. D.; Cragnolini, F.; Cenis, J. L.; Víllora, G. Production of Silk Fibroin Nanoparticles Using Ionic Liquids and High-Power Ultrasounds. J. Appl. Polym. Sci. 2015, 132, 41702. DOI: 10.1002/app.41702.

(5) Holland, C.; Numata, K.; Rnjak-Kovacina, J.; Seib, F. P. The Biomedical Use of Silk: Past, Present, Future. Adv. Healthcare Mater. 2019, 8, 1800465. DOI: 10.1002/adhm.201800465.

(6) Xiao, L.; Lu, G.; Lu, Q.; Kaplan, D. L. Direct Formation of Silk Nanoparticles for Drug Delivery. ACS Biomater. Sci. Eng. 2016, 2, 2050-2057. DOI: 10.1021/acsbiomaterials.6b00457.

(7) Mehrotra, S.; Chouhan, D.; Konwarh, R.; Kumar, M.; Jadi, P. K.; Mandal, B. B. Comprehensive Review on Silk at Nanoscale for Regenerative Medicine and Allied Applications. ACS Biomater. Sci. Eng. 2019, 5, 2054-2078. DOI: 10.1021/acsbiomaterials.8b01560.

(8) Wenk, E.; Merkle, H. P.; Meinel, L. Silk Fibroin as a Vehicle for Drug Delivery Applications. J. Controlled Release 2011, 150, 128-141. DOI: 10.1016/j.jconrel.2010.11.007.

(9) Tomeh, M. A.; Hadianamrei, R.; Zhao, X. Silk Fibroin as a Functional Biomaterial for Drug and Gene Delivery. Pharmaceutics 2019, 11, 494. DOI: 10.3390/pharmaceutics11100494.

(10) Jin, H.-J.; Kaplan, Mechanism of Silk Processing in Insects and Spiders. Nature 2003, 424, 1057-1061. DOI: 10.1038/nature01809. 
(11) Wongpinyochit, T.; Johnston, B. F.; Seib, F. P. Manufacture and Drug Delivery Applications of Silk Nanoparticles. J. Visualized Exp. 2016, e54669. DOI: 10.3791/54669.

(12) Wongpinyochit, T.; Totten, J. D.; Johnston, B. F.; Seib, F. P. Microfluidic-Assisted Silk Nanoparticle Tuning. Nanoscale Adv. 2019, 1, 873-883. DOI: 10.1039/c8na00208h.

(13) Solomun, J. I.; Totten, J. D.; Wongpinyochit, T.; Florence, A. J.; Seib, F. P. Manual versus Microfluidic-Assisted Nanoparticle Manufacture : Impact of Silk Fibroin Stock on Nanoparticle Characteristics. ACS Biomater. Sci. Eng. 2020, 6, 2796-2804. DOI: 10.1021/acsbiomaterials.0c00202.

(14) Dunderdale, G. J.; Davidson, S. J.; Ryan, A. J.; Mykhaylyk, O. O. Flow-Induced Crystallisation of Polymers from Aqueous Solution. Nat. Commun. 2020, 11, 3372. DOI: 10.1038/s41467-020-17167-8.

(15) McNeil, S. E. Nanoparticle Therapeutics: A Personal Perspective. Wiley Interdiscip. Rev.: Nanomed. and Nanobiotechnol. 2009, 1, 264-271. DOI: 10.1002/wnan.6.

(16) Wacker, M. Nanocarriers for Intravenous Injection - The Long Hard Road to the Market. Int. J. Pharm. 2013, 457, 50-62. DOI: 10.1016/j.ijpharm.2013.08.079.

(17) Lu, Q.; Zhu, H.; Zhang, C.; Zhang, F.; Zhang, B.; Kaplan, D. L. Silk Self-Assembly Mechanisms and Control From Thermodynamics to Kinetics. Biomacromolecules 2012, 13, 826832. DOI: $10.1021 / \mathrm{bm} 201731 \mathrm{e}$.

(18) Seib, F. P. Silk Nanoparticles-an Emerging Anticancer Nanomedicine. AIMS Bioeng. 2017, 4, 239-258. DOI: 10.3934/bioeng.2017.2.239. 
(19) Wenk, E.; Wandrey, A. J.; Merkle, H. P.; Meinel, L. Silk Fibroin Spheres as a Platform for Controlled Drug Delivery. J. Controlled Release 2008, 132, 26-34. DOI: 10.1016/j.jconrel.2008.08.005.

(20) Seib, F. P.; Jones, G. T.; Rnjak-Kovacina, J.; Lin, Y.; Kaplan, D. L. pH-Dependent Anticancer Drug Release from Silk Nanoparticles. Adv. Healthcare Mater. 2013, 2, 1606-1611. DOI: 10.1002/adhm.201300034.

(21) Subia, B.; Chandra, S.; Talukdar, S.; Kundu, S. C. Folate Conjugated Silk Fibroin Nanocarriers for Targeted Drug Delivery. Integr. Biol. 2014, 6, 203. DOI: 10.1039/c3ib40184g.

(22) Wongpinyochit, T.; Uhlmann, P.; Urquhart, A. J.; Seib, F. P. PEGylated Silk Nanoparticles for Anticancer Drug Delivery. Biomacromolecules 2015, 16, 3712-3722. DOI: 10.1021/acs.biomac.5b01003.

(23) Totten, J. D.; Wongpinyochit, T.; Carrola, J.; Duarte, I. F.; Seib, F. P. PEGylationDependent Metabolic Rewiring of Macrophages with Silk Fibroin Nanoparticles. ACS Appl. Mater. Interfaces 2019, 11, 14515-14525. DOI: 10.1021/acsami.8b18716.

(24) Crivelli, B.; Perteghella, S.; Bari, E.; Sorrenti, M.; Tripodo, G.; Chlapanidas, T.; Torre, M. L. Silk Nanoparticles: From Inert Supports to Bioactive Natural Carriers for Drug Delivery. Soft Matter 2018, 14, 546-557. DOI: 10.1039/c7sm01631.

(25) Gupta, V.; Aseh, A.; Ríos, C. N.; Aggarwal, B. B.; Mathur, A. B. Fabrication and Characterization of Silk Fibroin-Derived Curcumin Nanoparticles for Cancer Therapy. Int. J. Nanomed. 2009, 4, 115-122. DOI: 10.2147/ijn.s5581. 
(26) Zhao, Z.; Li, Y.; Xie, M.-B. Silk Fibroin-Based Nanoparticles for Drug Delivery. Int. J. Mol. Sci. 2015, 16, 4880-4903. DOI: 10.3390/ijms16034880.

(27) Gholami, A.; Tavanai, H.; Moradi, A. R. Production of Fibroin Nanopowder through Electrospraying. J. Nanopart. Res. 2011, 13, 2089-2098. DOI: 10.1007/s11051-010-9965-7.

(28) Toprakcioglu, Z.; Challa, P. K.; Morse, D. B.; Knowles, T. Attoliter Protein Nanogels from Droplet Nanofluidics for Intracellular Delivery. Sci. Adv. 2020, 6, eaay7952. DOI: 10.1126/sciadv.aay7952.

(29) Kazemimostaghim, M.; Rajkhowa, R.; Wang, X. Drug Loading and Release Studies for Milled Silk Particles of Different Sizes. Powder Technol. 2015, 283, 321-327. DOI: 10.1016/j.powtec.2015.05.042.

(30) Lu, Q.; Huang, Y.; Li, M.; Zuo, B.; Lu, S.; Wang, J.; Zhu, H.; Kaplan, D. L. Silk Fibroin Electrogelation Mechanisms. Acta Biomater. 2011, 7, 2394-2400. DOI: 10.1016/j.actbio.2011.02.032.

(31) Tarhini, M.; Greige-Gerges, H.; Elaissari, A. Protein-Based Nanoparticles: From Preparation to Encapsulation of Active Molecules. Int. J. Pharm. 2017, 522, 172-197. DOI: 10.1016/j.ijpharm.2017.01.067.

(32) Lepeltier, E.; Bourgaux, C.; Couvreur, P. Nanoprecipitation and the "Ouzo Effect": Application to Drug Delivery Devices. Adv. Drug Delivery Rev. 2014, 71, 86-97. DOI: 10.1016/j.addr.2013.12.009.

(33) Botet, R.; Roger, K. How Do Interactions Control Droplet Size during Nanoprecipitation? Curr. Opin. Colloid Interface Sci. 2016, 22, 108-112. DOI: 10.1016/j.cocis.2016.03.003. 
(34) Rode García, T.; García Ac, A.; Lalloz, A.; Lacasse, F-X.; Hildgen, P.; Rabanel, J-M.; Banquy, X. Unified Scaling of the Structure and Loading of Nanoparticles Formed by DiffusionLimited Coalescence. Langmuir 2018, 34, 5772-5780. DOI: 10.1021/acs.langmuir.8b00652.

(35) Agrahari, V.; Agrahari, V. Facilitating the Translation of Nanomedicines to a Clinical Product: Challenges and Opportunities. Drug Discov. Today 2018, 23, 974-991. DOI: 10.1016/j.drudis.2018.01.047.

(36) Pham, D. T.; Saelim, N.; Tiyaboonchai, W. Design of Experiments Model for the Optimization of Silk Fibroin Based Nanoparticles. Int. J. Appl. Pharm. 2018, 10, 195-201. DOI: 10.22159/ijap.2018v10i5.28139.

(37) Breslauer, D. N.; Muller, S. J.; Lee, L. P. Generation of Monodisperse Silk Microspheres Prepared with Microfluidics. Biomacromolecules 2010, 11, 643-647. DOI: 10.1021/bm901209u.

(38) Shimanovich, U.; Ruggeri, F. S.; De Genst, E.; Adamcik, J.; Barros, T. P.; Porter, D.; Müller, T.; Mezzenga, R.; Dobson, C. M.; Vollrath, F.; Holland, C.; Knowles, T. P. J. Silk Micrococoons for Protein Stabilisation and Molecular Encapsulation. Nat. Commun. 2017, 8, 15902. DOI: $10.1038 /$ ncomms 15902.

(39) Toprakcioglu, Z.; Levin, A.; Knowles, T. P. J. Hierarchical Biomolecular Emulsions Using 3-D Microfluidics with Uniform Surface Chemistry. Biomacromolecules 2017, 18, 3642-3651. DOI: $10.1021 /$ acs.biomac.7b01159.

(40) Kee, S. P.; Gavriilidis, A. Design and Characterisation of the Staggered Herringbone Mixer. Chem. Eng. J. 2008, 142, 109-121. DOI: 10.1016/j.cej.2008.02.001. 
(41) Matthew, S. A. L.; Totten, J. D.; Phuagkhaopong, S.; Egan, G.; Witte, K.; Perrie, Y.; Seib, F. P. Silk Nanoparticle Manufacture in Semi-Batch Format. ACS Biomater. Sci. Eng. 2020. DOI: 10.1021/acsbiomaterials.0c01028.

(42) Nisal, A.; Kalelkar, C.; Bellare, J.; Lele, A. Rheology and Microstructural Studies of Regenerated Silk Fibroin Solutions. Rheol. Acta 2013, 52, 833-840. DOI: 10.1007/s00397-0130723-5.

(43) Ianovska, M. A.; Mulder, P. P. M. F. A.; Verpoorte, E. Development of Small-Volume, Microfluidic Chaotic Mixers for Future Application in Two-Dimensional Liquid Chromatography. RSC Adv. 2017, 7, 9090-9099. DOI: 10.1039/c6ra28626g.

(44) Melton, L. A.; Lipp, C. W.; Spradling, R. W.; Paulson, K. A. DISMT - Determination of Mixing Time through Color Changes. Chem. Eng. Comm. 2002, 189, 322-338. DOI: $10.1080 / 00986440212077$.

(45) Weheliye, W.; Rodriguez, G.; Anderlei, T.; Micheletti, M.; Yianneskis, M.; Ducci, A. Appraisal of Shaken Bioreactor Mixing Efficiency for Different Operating Conditions. In Proceedings of the $14^{\text {th }}$ European conference on mixing Warszawa, Warsaw, Poland, 10-13 September 2012, pp. 503-508.

(46) Rodriguez, G.; Weheliye, W.; Anderlei, T.; Micheletti, M.; Yianneskis, M.; Ducci, A. Mixing Time and Kinetic Energy Measurements in a Shaken Cylindrical Bioreactor. Chem. Eng. Res. Des. 2013, 91, 2084-2097. DOI: 10.1016/j.cherd.2013.03.005.

(47) Tomar, S. Converting Video Formats with FFmpeg. Linux Journal 2006, 146, 10. 
(48) Rodriguez, G.; Anderlei, T.; Micheletti, M.; Yianneskis, M.; Ducci, A. On the Measurement and Scaling of Mixing Time in Orbitally Shaken Bioreactors. Biochem. Eng. J. 2014, 82, 10-21. DOI: 10.1016/j.bej.2013.10.021.

(49) Xu, Z.; Lu, C.; Riordon, J.; Sinton, D.; Moffitt, M. G. Microfluidic Manufacturing of Polymeric Nanoparticles: Comparing Flow Control of Multiscale Structure in Single-Phase Staggered Herringbone and Two-Phase Reactors. Langmuir 2016, 32, 12781-12789. DOI: 10.1021/acs.langmuir.6b03243.

(50) ASTM E691-19e1. Standard Practice for Conducting an Interlaboratory Study to Determine the Precision of a Test Method; West Conshohocken, CA, 2019. DOI: 10.1520/E0691$19 \mathrm{E} 01$.

(51) Pang, F.-M.; Seng, C.-E.; Teng, T.-T; Ibrahim, M. H. Densities and Viscosities of Aqueous Solutions of 1-Propanol and 2-Propanol at Temperatures from 293.15 K to 333.15 K. J. Mol. Liq. 2007, 136, 71-78. DOI: 10.1016/j.molliq.2007.01.003.

(52) Williams, M. S.; Longmuir, K. J.; Yager, P. A Practical Guide to the Staggered Herringbone Mixer. Lab Chip 2008, 8, 1121-1129. DOI: 10.1039/b802562b.

(53) Mialdun, A.; Yasnou, V.; Shevtsova, V.; Königer, A.; Köhler, W.; Alonso De Mezquia, D.; Bou-Ali, M. M. A Comprehensive Study of Diffusion, Thermodiffusion, and Soret Coefficients of Water-Isopropanol Mixtures. J. Chem. Phys. 2012, 136, 244512. DOI: 10.1063/1.4730306. 
(54) Son, Y. Determination of Shear Viscosity and Shear Rate from Pressure Drop and Flow

Rate Relationship in a Rectangular Channel. Polymer 2007, 48, 632-637. DOI: 10.1016/j.polymer.2006.11.048.

(55) Yang, H.; Yang, S.; Kong, J.; Dong, A.; Yu, S. Obtaining Information about Protein Secondary Structures in Aqueous Solution Using Fourier Transform IR Spectroscopy. Nat. Protoc. 2015, 10, 382-396. DOI: 10.1038/nprot.2015.024.

(56) Hu, X.; Kaplan, D.; Cebe, P. Determining Beta-Sheet Crystallinity in Fibrous Proteins by Thermal Analysis and Infrared Spectroscopy. Macromolecules 2006, 39, 6161-6170. DOI: 10.1021/ma0610109.

(57) Griebenow, K.; Santos, A. M.; Carrasquillo, K. G. Secondary Structure of Proteins in the Amorphous Dehydrated State Probed by FTIR Spectroscopy. Dehydration-Induced Structural Changes and Their Prevention. Internet J.Vib.Spectrosc. 1999, 3, 1-2.

(58) Riesen, R.; Vogel, K.; Schubnell, M. DSC by the TGA/SDTA851e Considering Mass Changes. J. Therm. Anal. Calorim. 2001, 64, 243-252. DOI: 10.1023/A:1011505617274.

(59) Galisteo-González, F.; Molina-Bolívar, J. A. Systematic Study on the Preparation of BSA Nanoparticles. Colloids Surf., B 2014, 123, 286-292. DOI: 10.1016/j.colsurfb.2014.09.028.

(60) Galindo-Rodríguez, S. A.; Puel, F.; Briançon, S.; Allémann, E.; Doelker, E.; Fessi, H. Comparative Scale-up of Three Methods for Producing Ibuprofen-Loaded Nanoparticles. Eur. J. Pharm. Sci. 2005, 25, 357-367. DOI: 10.1016/j.ejps.2005.03.013.

(61) Zhang, Y.; Zuo, Y.; Wen, S.; Hu, Y.; Min, Y. Distinctive Stress-Stiffening Responses of Regenerated Silk Fibroin Protein Polymers under Nanoscale Gap Geometries: Effect of Shear on 
Silk Fibroin-Based Materials. Biomacromolecules 2018, 19, 1223-1233. DOI: 10.1021/acs.biomac.8b00070.

(62) Joye, I. J.; McClements, D. J. Production of Nanoparticles by Anti-Solvent Precipitation for Use in Food Systems. Trends Food Sci. Technol. 2013, 34, 109-123. DOI: 10.1016/j.tifs.2013.10.002.

(63) Um, I. C.; Kweon, H. Y.; Park, Y. H.; Hudson, S. Structural Characteristics and Properties of the Regenerated Silk Fibroin Prepared from Formic Acid. Int. J. Biol. Macromol. 2001, 29, 9197. DOI: 10.1016/S0141-8130(01)00159-3.

(64) Webb, C.; Forbes, N.; Roces, C. B.; Anderluzzi, G.; Lou, G.; Abraham, S.; Ingalls, L.; Marshall, K.; Leaver, T. J.; Watts, J. A.; Aylott, J. W.; Perrie, Y. Using Microfluidics for Scalable Manufacturing of Nanomedicines from Bench to GMP: A Case Study Using Protein-Loaded Liposomes. Int. J. Pharm. 2020, 582, 119266. DOI: 10.1016/j.ijpharm.2020.119266.

(65) Matsumoto, A.; Lindsay, A.; Abedian, B.; Kaplan, D. L. Silk Fibroin Solution Properties Related to Assembly and Structure. Macromol. Biosci. 2008, 8, 1006-1018. DOI: 10.1002/mabi.200800020.

(66) Zainuddin; Le, T. T.; Park, Y.; Chirila, T. V.; Halley, P. J.; Whittaker, A. K. The Behavior of Aged Regenerated Bombyx Mori Silk Fibroin Solutions Studied by ${ }^{1} \mathrm{H}$ NMR and Rheology. Biomaterials 2008, 29, 4268-4274. DOI: 10.1016/j.biomaterials.2008.07.041. 
(67) Chung, D. E.; Um, I. C. Effect of Molecular Weight and Concentration on Crystallinity and Post Drawing of Wet Spun Silk Fibroin Fiber. Fibers Polym. 2014, 15, 153-160. DOI: 10.1007/s12221-014-0153-8. 